\title{
Goal-Directed Weakening of Abstract Interpretation Results
}

\author{
SUNAE SEO \\ Korea Advanced Institute of Science and Technology \\ HONGSEOK YANG and KWANGKEUN YI \\ Seoul National University \\ and \\ TAISOOK HAN \\ Korea Advanced Institute of Science and Technology
}

One proposal for automatic construction of proofs about programs is to combine Hoare logic and abstract interpretation. Constructing proofs is in Hoare logic. Discovering programs' invariants is done by abstract interpreters.

One problem of this approach is that abstract interpreters often compute invariants that are not needed for the proof goal. The reason is that the abstract interpreter does not know what the proof goal is, so it simply tries to find as strong invariants as possible. These unnecessary invariants increase the size of the constructed proofs. Unless the proof-construction phase is notified which invariants are not needed, it blindly proves all the computed invariants.

In this article, we present a framework for designing algorithms, called abstract-value slicers, that slice out unnecessary invariants from the results of forward abstract interpretation. The framework provides a generic abstract-value slicer that can be instantiated into a slicer for a particular abstract interpretation. Such an instantiated abstract-value slicer works as a postprocessor to an abstract interpretation in the whole proof-construction process, and notifies to the

S. Seo and T. Han were supported by Korea Ministry of Information and Communication under the Information Technology Research Center support program, supervised by the Institute of Information Technology Assessment (IITA-2005-C1090-0502-0031). H. Yang was supported by EPSRC and the Basic Research Program of the Korea-Science \& Engineering Foundation (grant No. R082003-000-10370-0). K. Yi was supported by Brain Korea 21 Project of Korea Ministry of Education and Human Resources, by IT Leading R\&D Support Project of Korea Ministry of Information and Communication, by Korea Research Foundation grant KRF-2003-041-D00528, and by National Security Research Institute of Korea.

Authors' addresses: S. Seo and T. Han, Department of Computer Science, Korea Advanced Institute of Science and Technology 373-1 Guseong-dong Yuseong-gu Daejeon 305-701, Korea; email: \{saseo,han\}@pllab.kaist.ac.kr; H. Yang, Department of Computer Science, Queen Mary, University of London, Mile End Road London E1 4NS UK; email: hyang@dcs.qmul.ac.uk; K. Yi, School of Computer Science and Engineering, Seoul National University, San 56-1 Shilim-dong Gwanak-gu Seoul 151-744 Korea; email: kwang@ropas.snu.ac.kr.

Permission to make digital or hard copies of part or all of this work for personal or classroom use is granted without fee provided that copies are not made or distributed for profit or direct commercial advantage and that copies show this notice on the first page or initial screen of a display along with the full citation. Copyrights for components of this work owned by others than ACM must be honored. Abstracting with credit is permitted. To copy otherwise, to republish, to post on servers, to redistribute to lists, or to use any component of this work in other works requires prior specific permission and/or a fee. Permissions may be requested from Publications Dept., ACM, Inc., 2 Penn Plaza, Suite 701, New York, NY 10121-0701 USA, fax +1 (212) 869-0481, or permission@acm.org. (C) 2007 ACM 0164-0925/2007/10-ART39 \$5.00 DOI 10.1145/1286821.1286830 http://doi.acm.org/ $10.1145 / 1286821.1286830$

ACM Transactions on Programming Languages and Systems, Vol. 29, No. 6, Article 39, Publication date: October 2007. 
next proof-construction phase which invariants it does not have to prove. Using the framework, we designed an abstract-value slicer for an existing relational analysis and applied it on programs. In this experiment, the slicer identified $62 \%-81 \%$ of the computed invariants as unnecessary, and resulted in $52 \%-84 \%$ reduction in the size of constructed proofs.

Categories and Subject Descriptors: F.3.1 [Logics and Meanings of Programs]: Specifying and Verifying and Reasoning about Programs-Mechanical verification; D.2.4 [Software Engineering]: Software/Program Verification-Correctness proofs, Formal methods; D.3.1 [Programming Languages]: Formal Definitions and Theory-Semantics

General Terms: Algorithms, Design, Languages, Verification

Additional Key Words and Phrases: Abstract interpretation, backward analysis, Hoare logic, program verification, static analysis

\section{ACM Reference Format:}

Seo, S., Yang, H., Yi, K., and Han, T. 2007. Goal-directed weakening of abstract interpretation results. ACM Trans. Program. Lang. Syst. 29, 6, Article 39 (October 2007), 39 pages. DOI = 10.1145/1286821.1286830 http://doi.acm.org/ 10.1145/1286821.1286830

\section{INTRODUCTION}

Though Proof-Carrying Code (PCC) technologies [Necula and Schneck 2002; Necula and Rahul 2001; Appel 2001; Hamid et al. 2002] have been a convincing approach for certifying the safety of code, how to achieve the code's safety proof is still a matter for investigation. The existing proof construction process is either not fully automatic, assuming that the program invariants should be provided by the programmer [Necula 1997; Necula and Lee 1997; Necula and Rahul 2001], or limited to a class of properties that are automatically inferrable by the current type system technologies [Hamid et al. 2002; Appel and Felty 2000; Morrisett et al. 1998].

One proposal [Seo et al. 2003] for automatic construction of proofs for a wide class of program properties is to combine abstract interpretation [Cousot and Cousot 1977; Cousot 1998] and Hoare logic [Hoare 1969]. Constructing proofs is in Hoare logic. Discovering program's invariants, which is the main challenge in automatically constructing Hoare proofs, is done by abstract interpreters [Cousot and Cousot 1977; Cousot 1998]. An abstract interpreter estimates program properties (i.e., approximate invariants) of interest, and the proof-construction method constructs a Hoare proof for those approximate invariants. The gap between the estimated invariants of an abstract interpreter and the preconditions "computed by Hoare-logic proof rules" is filled by the soundness of the abstract interpreter only, without involving any theorem provers. For instance, when the abstract interpreter's results (i.e., approximate invariants at program points-boxed properties here) are $\mathrm{p} x:=E \mathrm{q}$, the soundness proofs of the abstract interpreter are used to produce a proof that $p$ implies the weakest precondition of $x:=E$ for $\mathrm{q}$.

This proof-construction method for PCC has several appealing features. Once designed, an abstract interpreter can be used repeatedly for all programs of the target language, as long as their properties to verify and check remain the same. Furthermore, the proof-checking side (code consumer's side) is insensitive to a 
specific abstract interpreter. The code consumer does not have to know which analysis technique has been used to generate the proof. The assertion language in Hoare logic is fixed to first-order logic for integers, into which we have to translate abstract interpretation results. This translation procedure is defined by referencing the concretization formulas of the used abstract interpreter. Lastly, the proof-checking side is simple. Checking the Hoare proofs is simply by pattern-matching the proof tree nodes against the corresponding Hoare logic rules. Checking if the proofs are about the accompanied code is straightforward, because the program texts are embedded in the Hoare proofs.

\subsection{Problem}

This work is motivated by one problem in the proof-construction method: abstract interpretation results are often unnecessarily informative for intended Hoare proofs. A (forward) abstract interpreter is usually designed to compute (approximate) program invariants that are as strong as possible, so that the computed invariants can verify a wide class of safety properties. Thus, when the abstract interpreter is used to verify one specific safety property, its results usually contain some (approximate) program invariants that are not necessary to prove the safety property of interest, although those invariants might be needed for some other safety properties. For instance, in our experiment with an existing relational analysis [Miné 2001], $62 \%-81 \%$ of the analysis results were not needed for the intended verification. ${ }^{1}$

The existence of such unnecessary invariants among the results of an abstract interpretation becomes a bottleneck for all the efforts to reduce the proof size. When a Hoare proof of a safety property is constructed from the abstract interpretation results, it consists of two kinds of subproofs: the ones that the abstract interpretation results are indeed (approximate) invariants, and the others that those approximate invariants imply the safety property. The unnecessarily informative analysis results mainly cause the first kind of subproofs to "explode"; they increase the number of such subproofs, by adding useless proof subgoals.

Without addressing this problem, the proof-construction method often produces unnecessarily big Hoare proofs, and hence is likely to be impractical for PCC. Big proofs accompanying mobile code degrade the code mobility in a network that usually has a limited bandwidth, or are impractical for code consumers that usually are small embedded systems with a limited memory. Note that no techniques for representing subproofs compactly by some clever encoding can solve the problem, because they assume that all subproofs are necessary; it is not the purpose of such techniques to identify the useless subproofs.

Example 1.1. As an example where abstract interpretation results are stronger than necessary, consider the following assignment sequence with the parity abstract interpretation, which estimates whether each program variable contains an even integer or an odd integer:

$$
\mathrm{x}:=4 \mathrm{x} ; \mathrm{x}:=2 \mathrm{x} \text {. }
$$

${ }^{1}$ We explain this further in Section 5. 
The estimated invariants from the abstract interpretation for variable $\mathrm{x}$ are:

$$
\top \mathrm{x}:=4 \mathrm{x} ; \text { even } \mathrm{x}:=2 \mathrm{x} \text { even. }
$$

Suppose we are interested in constructing a proof that variable $x$ at the end is an even integer. Then the invariant "even" after the first assignment, which means $\mathrm{x}$ is an even integer, is stronger than needed; just $T$ is enough. This is because for the second assignment, Hoare triple $\{$ true $\} \mathrm{x}:=2 \mathrm{x}\{\exists n . x=2 n\}$ can be derived

$$
\frac{\overline{\text { true } \Rightarrow \exists n .2 x=2 n} \quad \overline{\{\exists n .2 x=2 n\} \mathrm{x}:=2 \mathrm{x}\{\exists n . x=2 n\}}}{\{\text { true }\} \mathrm{x}:=2 \mathrm{x}\{\exists n \cdot x=2 n\}} .
$$

and this triple is enough to construct the intended proof:

$$
\frac{\overline{\{\text { true }\} \mathrm{x}:=4 \mathrm{x}\{\text { true }\}} \overline{\{\text { true }\} \mathrm{x}:=2 \mathrm{x}\{\exists n . x=2 n\}}}{\{\text { true }\} \mathrm{x}:=4 \mathrm{x} ; \mathrm{x}:=2 \mathrm{x}\{\exists n . x=2 n\}} .
$$

That is, the following invariants, weaker than the original results, are just enough for our proof goal:

$$
\top \mathrm{x}:=4 \mathrm{x} ; \mathrm{T} \mathrm{x}:=2 \mathrm{x} \text { even. }
$$

Example 1.2. Similarly, as another example where useless invariants occur in the results of an abstract interpretation, consider the following program, again with the parity abstract interpretation.

$$
\mathrm{x}:=1 ; \mathrm{y}:=2 \mathrm{x} .
$$

The estimated invariants from the abstract interpretation for each variable at each program point are as follows:

$$
x \mapsto \top, y \mapsto \top \quad \mathrm{x}:=1 ; \quad x \mapsto \text { odd, } y \mapsto \top \quad \mathrm{y}:=2 \mathrm{x} \quad x \mapsto \text { odd, } y \mapsto \text { even } .
$$

Suppose we are interested in constructing a proof that variable y at the end is an even integer. Then, all invariants about $\mathrm{x}$ are useless. Just $T$ for $\mathrm{x}$ is enough to reach the proof goal:

$$
\frac{\overline{\{\text { true }\} \mathrm{x}:=1\{\text { true }\}} \frac{\overline{\text { true } \Rightarrow(\exists n .2 x=2 n)} \overline{\{\exists n .2 x=2 n\} \mathrm{y}:=2 \mathrm{x}\{\exists n \cdot y=2 n\}}}{\{\text { true }\} \mathrm{y}:=2 \mathrm{x}\{\exists n \cdot y=2 n\}}}{\{\text { true }\} \mathrm{x}:=1 ; \mathrm{y}:=2 \mathrm{x}\{\exists n \cdot y=2 n\}}
$$

Thus, the original analysis results can be weakened to the following, while still proving that $\mathrm{y}$ is even at the end:

$$
x \mapsto \top, y \mapsto \top \quad \mathrm{x}:=1 ; \quad x \mapsto \top, y \mapsto \top \quad \mathrm{y}:=2 \mathrm{x} ; \quad x \mapsto \top, y \mapsto \text { even } .
$$

This example illustrates that the conventional program slicing technique does not immediately provide a satisfactory solution for our problem. One naive idea to eliminate the unnecessary information from the abstract interpretation result is to apply first the program slicing and then an abstract interpretation. However, for this example, this approach cannot identify any useless information from the abstract interpretation result. When the program slicing is applied to the example program with the slicing criterion "the value of variable $\mathrm{y}$ after $\mathrm{y}:=2 \mathrm{x}$," it cannot slice out any parts of the program, because both $\mathrm{x}:=1$ 




(a) Before slicing

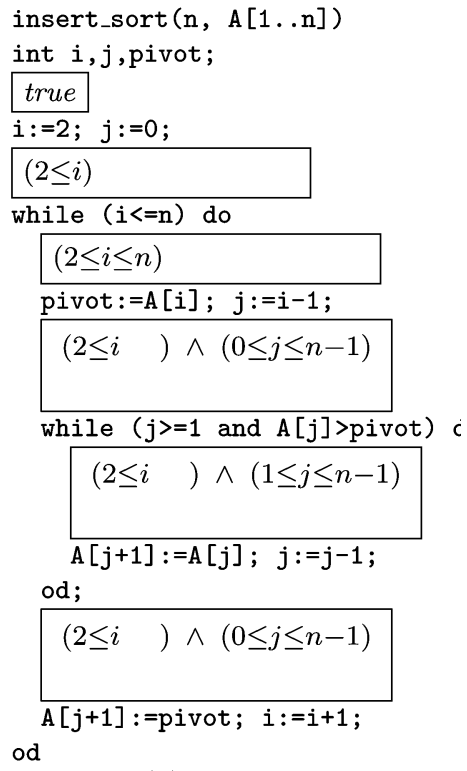

(b) After slicing

Fig. 1. Annotated insertion sort before and after slicing.

and $\mathrm{y}:=2 \mathrm{x}$ affect the slicing criterion. As a result, the following parity abstract interpretation is given the unmodified original program, thus getting no help from the program slicing. Another idea might be to use dependency analysis in program slicing; to compute the dependency relationship between variables at different program points, and then to use this relationship to slice the abstract interpretation result. When this idea is applied to our example, it finds out that $x \mapsto$ odd after $\mathrm{y}:=2 \mathrm{x}$ is not necessary, but it fails to discover that $x \mapsto$ odd after $x:=1$ is not needed for verification. Given the slicing criterion "the value of variable y after $y:=2 x$, dependency analysis finds out that the value of variable y after $\mathrm{y}:=2 \mathrm{x}$ is dependent upon that of variable $\mathrm{x}$ after $\mathrm{x}:=1$. Thus, the following slicing step does not delete $x \mapsto$ odd after $\mathrm{x}:=1$.

Example 1.3. To see the problem in a "real world," we consider a slightly more realistic program - the insertion sort. Figure 1(a) shows the insertion sort, which is annotated with results of an abstract interpreter named "zone analysis" [Miné 2001]. Zone analysis estimates the upper and lower bounds of expressions $x$ and $x-y$, for all program variables $x$ and $y$. The insertion sort program takes an array A and the size $\mathrm{n}$ of the array as an input, and sorts the array.

Suppose that we ran the abstract interpreter in order to verify the absence of array index errors in the program. The annotations in the program prove this safety property. ${ }^{2}$

However, note that the annotations also contain unnecessary information. For instance, $i \leq n$ in the annotation marked by $*$ neither is helpful for showing

${ }^{2}$ Here we assume that in " $B_{1}$ and $B_{2}$, " $B_{2}$ is evaluated only when $B_{1}$ is true. 
that the subsequent array access $A[j+1]$ is within bounds, nor is used to imply the loop invariant $(2 \leq i) \wedge(0 \leq j \leq i+2)$. Thus, it can be eliminated without breaking the proof. In fact, half of the annotations in the program are not needed. Figure 1 shows the program where all such useless invariants are eliminated.

\subsection{Our Solution}

In this article, we present an algorithm, called abstract-value slicer, that filters out unnecessary invariants from the results of a forward abstract interpreter. The abstract-value slicer works as a post-processor to the abstract interpreter. Given an annotated program and a property of interest, the slicer approximates all the annotations further, until all the information in each annotation contributes to the verification of the property.

The main idea of the abstract-value slicer is to view an abstract interpretation result at each program point as conjunction of formulas, and to find out which formulas in the conjunction are not necessary for verification. For instance, suppose that an abstract interpreter analyzed the assignment $x:=E$ for an input abstract value that means $p_{1} \wedge p_{2} \wedge p_{3}$, and that it produced an output abstract value that means $p_{1}^{\prime} \wedge p_{2}^{\prime}$. That is, the abstract interpreter verified that if a pre state satisfies $p_{1} \wedge p_{2} \wedge p_{3}$, then the post state after the assignment satisfies $p_{1}^{\prime} \wedge p_{2}^{\prime}$. When the abstract-value slicer is given this analysis result and it is notified that only $p_{1}^{\prime}$ is used for verification, the slicer computes a subset $P \subseteq\left\{p_{1}, p_{2}, p_{3}\right\}$ such that (1) $\wedge P$ can be represented by some abstract value and (2) although $\wedge P$ is weaker than the original $p_{1} \wedge p_{2} \wedge p_{3}$, it is still strong enough to ensure that the assignment can achieve the goal $p_{1}^{\prime}$ : if the pre state satisfies $\wedge P$, then the post state of the assignment satisfies $p_{1}^{\prime}$. Then, the slicer filters out the formulas in $\left\{p_{1}, p_{2}, p_{3}\right\}-P$ that are not necessary for verification: the slicer replaces the original input abstract value " $\left\{p_{1}, p_{2}, p_{3}\right\}$ " by the abstract state that means $\wedge P$.

A reader might feel that a better alternative approach for solving the problem of unnecessary invariants is to use "on-line," goal-oriented backward abstract interpreters that compute the underapproximation of the weakest precondition, that is, a set of pre states from which a program always achieves the given goal. Note that our approach is, on the other hand, in the reverse direction and "off-line" yet achieving the same effect. Given the results of forward abstract interpreters, which are already underapproximations of the weakest preconditions, we weaken the underapproximate results and make them closer from below to the weakest preconditions. Please be reminded that our problem is to underapproximate the weakest preconditions under which a program must always satisfy the given goal property.

Although designing such an abstract interpreter (an underapproximate backward precondition analyzer) is plausible, we pursue the idea of designing an abstract-value slicer over an existing forward abstract interpreter. That is, such an abstract-value slicer is not a new analysis for estimating goal-directed invariants but an "off-line" method that reuses the results of existing abstract interpreters to achieve goal-directed invariants. 
This separation (or, modularization) of the slicing from the analysis is meaningful for the reuse of the analysis. First, the analysis results can be reused. Computed analysis results for a program can be reused to achieve different slices for different slicing criteria (proof goals). The analysis itself can be reused too. For example, once an abstract interpreter is designed originally for detecting buffer-overrun errors by estimating the ranges of buffer-accessing indexes, it can be reused now to provide our slicer with invariant candidates for being used in buffer-overrun nonexistence proofs. We consider forward abstract interpreters because they are most common in design and practice, having a number of realistic instances. Our method can be seen as a method for achieving goaldirected analysis results from the results of a usual, goal-independent, forward abstract interpretation.

The contributions of the article are:

-We present a general framework for designing correct abstract-value slicers. The framework defines the generic abstract-value slicer, which we can instantiate into a specific slicer for a particular abstract interpreter, by providing parameters. The framework also specifies the soundness conditions for those parameters of the generic slicer; if the parameters satisfy these conditions, the resulting slicer filters out only the unnecessary parts from abstract interpretation results.

-We present methods for constructing parameters of the generic abstractvalue slicer, and show when all the constructed parameters by these methods satisfy the soundness conditions.

-Using our framework, we build a specific abstract-value slicer for zone analysis [Miné 2001], and demonstrate its effectiveness in the context of proof construction. In our experiment, the slicer identified that $62 \%-81 \%$ of the abstract interpretation results are not necessary for the verification, and resulted in 52\%-84\% reduction in the size of constructed program proofs.

\subsection{Related Work}

Our abstract-value slicer is closely related to program slicing [Tip 1995; Rival 2005a] and cone of influences [Clarke et al. 1999] in model checking. All these techniques, including ours, identify the irrelevant parts for achieving a given goal, and slice them out. The objects that get sliced are, however, different: the abstract-value slicer works only on the abstract interpretation results, while program slicing and cone of influence modify a program or a Kripke structure that models the behavior of a program.

Another important difference lies in the computation of the irrelevant parts for the goal. In order to detect the irrelevant parts, both program slicing techniques and cone of influences compute (an overapproximation of) the dependency between program variables at different program points. Intuitively, the computed dependency of $y$ at $l_{1}$ on $x$ at $l_{0}$ means that some concrete computation uses the value of $x$ at $l_{0}$ to compute the value of $y$ at $l_{1}$, so that the different values of $x$ at $l_{0}$ will make $y$ have different values at $l_{1}$. Recently, Rival [2005a] generalized this dependency in his abstract program 
slicing, so that the dependency is now between facts about one variable, such as $x>3$ and $y<9$, but it is still about the concrete computations. The abstract-value slicer, on the other hand, computes the dependency in the $a b$ stract computation between general facts which might involve multiple variables such as $x \leq z+3$ at $l_{0}$ and $z \leq y \leq z+9$ at $l_{1}$. For instance, suppose that an abstract interpretation result of the assignment $\mathrm{x}:=\mathrm{y}-\mathrm{z}$ is $(y \leq z+1 \wedge v \leq y) \wedge(y \leq v \wedge v \leq z+1) \quad \mathrm{x}:=\mathrm{y}-\mathrm{z} \quad x \leq 1 \wedge v \leq z+1$, and the abstract-value slicer is asked to check whether $x \leq 1$ in the post abstract value depends on the first conjunct $y \leq z+1 \wedge v \leq y$ of the pre abstract value. If by the same abstract interpretation the other conjunct can result in the same conclusion $x \leq 1$, that is, $y \leq v \wedge v \leq z+1 \mathrm{x}:=\mathrm{y}-\mathrm{z} x \leq 1$, then the abstract-value slicer reports that $x \leq 1$ does not depend on the first conjunct $y \leq z+1 \wedge v \leq y$. Otherwise, that is, if the abstract interpreter approximates too much that its result from $y \leq v \wedge v \leq z+1$ does not imply $x \leq 1$, then the slicer decides that $x \leq 1$ depends on $y \leq z+1 \wedge v \leq y$. This is so, although the Hoare triple $\{y \leq v \wedge v \leq z+1\} \mathrm{x}:=\mathrm{y}-\mathrm{z}\{x \leq 1\}$ holds in the concrete semantics and thus there is no such dependency in the concrete semantics.

The dependency between general facts is also considered in the work on the abstract noninterference [Giacobazzi and Mastroeni 2004]. However, unlike our abstract-value slicers, the dependency in the abstract noninterference is about the concrete computations, not about the abstract computations. Moreover, the existing work on the abstract noninterference does not consider the algorithm for computing the dependency relation, while the main focus of our work is to find such an algorithm.

Another related line of research is the work on abstraction refinement and predicate abstraction [Graf and Sä̈di 1997; Clarke et al. 2000; Ball and Rajamani 2001; Ball et al. 2001; Henzinger et al. 2003, 2002]. The analyzers [Ball and Rajamani 2001; Henzinger et al. 2003] based on these two techniques usually find an abstract domain that is as abstract as possible but still precise enough for verifying a particular property. However, for the problem of identifying unnecessary invariants, abstraction refinement and predicate abstraction are not widely applicable, because many existing abstract interpretations are not based on those techniques. Our abstract-value slicers, on the other hand, can be applied for such abstract interpretations. We note that the analyzers based on abstraction refinement and the abstract-value slicers work in opposite "directions." Such analyzers start with naive analysis results and keep strengthening the results until they are sufficient to prove a property of interest. On the other hand, the slicers start with precise analysis results, which already prove the property of interest, and weaken the results, while maintaining the proof.

From the view point that our off-line backward abstract-value slicing after an overapproximate forward post-condition analyzer can be simulated by a single underapproximate backward precondition analyzer, related works are backward abstract interpreters that underapproximate the weakest preconditions. Please note that, however, backward abstract interpreters in Rival [2005b], Cousot [2005], Cousot and Cousot [1999], Massé [2001], Hughes and 
Launchbury [1992], Duesterwald et al. [1995] and Bourdoncle [1993], overapproximate the weakest preconditions. ${ }^{3}$ Their backward abstract interpreters discover a superset of the pre states where a program might generate an error. Thus such backward abstract interpreters are used in program debugging [Bourdoncle 1993] and alarm inspection [Rival 2005b]. On the other hand, abstract model checkers [Dams et al. 1997] can be seen as backward abstract interpreters that underapproximate the weakest preconditions [Cousot 1981].

Projection analysis [Wadler and Hughes 1987; Hughes 1988; Davis and Wadler 1990] in functional programs and mode analyses [King and Lu 2002; Howe et al. 2004] in logic programs both underapproximate the weakest preconditions sharing the same goal as our abstract-value slicer, but their techniques are not directly applicable to the problem of this article. The projection analysis estimates a function that transforms the demand for the output to the one for the input. The demand for the output specifies which part of the output will be needed by the environment of the program (i.e., continuation), and the demand for the input expresses which property of the input is sufficient for the program to produce the necessary part of the output. Mode analysis in logic programs is similar. It estimates context properties that, if satisfied by the initial query, guarantee that the program with the query never generate any moding error. However, the domains used in these backward analyses are not as general as the ones used in our framework. Our abstract-value slicer works with complex domains that are infinite or relational, such as interval domain and zone domain [Cousot and Cousot 1977; Miné 2001], can have nontrivial domain operations (e.g., closure operation in zone domain), or can require an acceleration method (e.g., widening) for quick convergence.

\subsection{Organization}

We start the article by reviewing the basics of the abstract interpretations in Section 2. In Section 3, we define a generic abstract-value slicer parameterized by extractor domain and back-tracers for assignments and Boolean expressions. Intuitively, the extractor domain specifies the working space of the slicer, and the back-tracers describe how the slicer treats each assignments and Boolean expressions. In that section, we specify the soundness conditions for these two parameters, and prove that the conditions ensure the correctness of the instantiated slicer. In the next section, we present methods for constructing parameters to the slicer, which satisfy the soundness requirements. There we describe two techniques for constructing correct back-tracers. In Section 5, we explain the experimental results about one specific abstract-value slicer in the context of proof construction. Finally, we conclude the article in Section 6.

\footnotetext{
${ }^{3}$ This statement is true only for terminating deterministic programs, because those backward abstract interpreters overapproximate so-called pre state-sets. The pre state-set of a command $C$ for a postcondition $p$ is the set of pre states from which $C$ can output some state satisfying $p$. For terminating deterministic programs, the pre state-set of $C$ and $p$ is the same as the weakest precondition of $C$ and $p$.
}

ACM Transactions on Programming Languages and Systems, Vol. 29, No. 6, Article 39, Publication date: October 2007. 


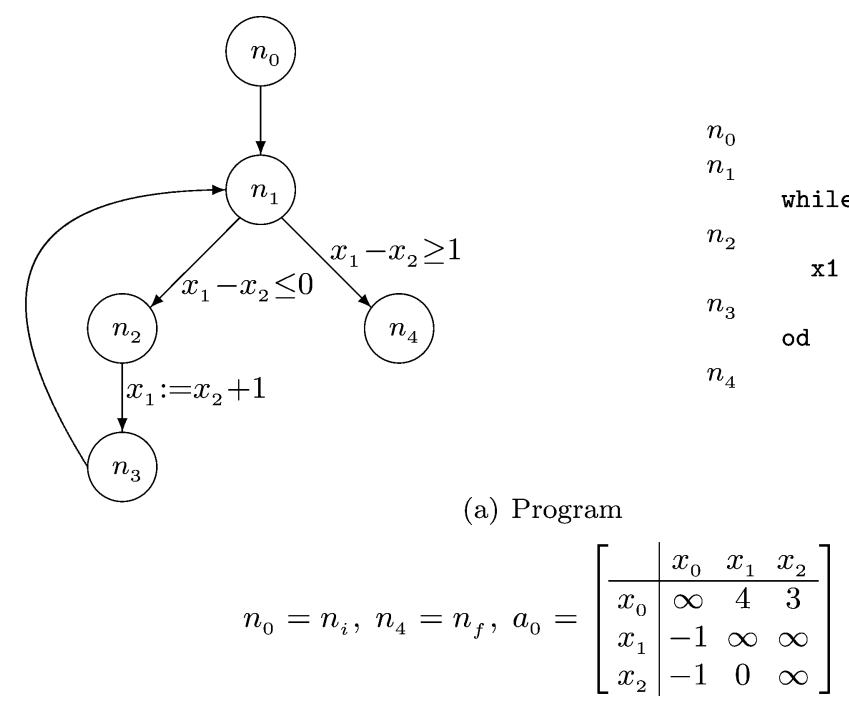

(b) Entry, exit node and initial abstract state

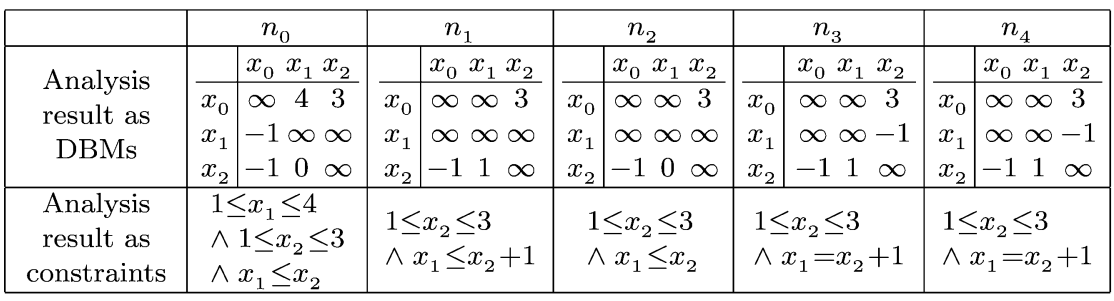

(c) Analysis result

Fig. 2. An example program and its abstract interpretation result from zone analysis.

\section{ABSTRACT INTERPRETATION}

We consider programs represented by control flow graphs [Cousot and Cousot 1977]. Let ATerm be the set of atomic terms, that is, all the inequalities $E \leq E^{\prime}$, assignments $x:=E$, and command skip. A program $\left(V, E, n_{i}, n_{f}, L\right)$ is a finite graph with nodes in $V$ and edges in $E$, together with two special nodes $n_{i}$ and $n_{f}$, and a labeling function $L: E \rightarrow$ ATerm. A node in $V$ represents a program point, and an edge in $E$ a flow of control between program points; with this flow, an inequality or assignment is associated, and the labeling function $L$ expresses this association. The special nodes $n_{i}$ and $n_{f}$, respectively, denote the entry and exit of the program. We assume that in the program, no edges go into the entry node $n_{i}$, and no edges come out of the exit node $n_{f}$. Figure 2 (a) shows a program that represents code with a single while loop. In this program, we label each edge with an atomic term, except when the atomic term is skip. Another thing to note is that the condition for exiting the loop, $\neg\left(x_{1}-x_{2} \leq 0\right)$, is expressed by an equivalent condition $x_{1}-x_{2} \geq 1$; these two conditions are equivalent since variables range over integers. 
In the article, we consider abstract interpretations that consist of three components: a join semilattice $\mathcal{A}=(A, \sqsubseteq, \perp, \sqcup)$, the abstract semantics ${ }^{4}$ $\llbracket-\rrbracket:$ ATerm $\rightarrow\left(\mathcal{A} \rightarrow_{m} \mathcal{A}\right)$ of atomic terms, and a strategy for computing post fixpoints. Given a program $\left(V, E, n_{i}, n_{f}, L\right)$ and an initial abstract state $a_{0} \in \mathcal{A}$, the abstract interpretation first uses $\mathcal{A}$ and $\llbracket-\rrbracket$ to define "abstract step function" $F$ :

$$
\begin{aligned}
& F: \prod_{n \in V} \mathcal{A} \rightarrow_{m} \prod_{n \in V} \mathcal{A} \\
& F(f)(n) \stackrel{\text { def }}{=} \begin{cases}a_{0} & \text { if } n=n_{i} \\
\bigsqcup\{\llbracket L(m n) \rrbracket(f(m)) \mid m n \in E\} & \text { otherwise. }\end{cases}
\end{aligned}
$$

Here $\prod_{n \in V} \mathcal{A}$ is the product join semilattice, which consists of tuples $f$ of elements in $\mathcal{A}$ and inherits the order structure from $\mathcal{A}$ pointwise. ${ }^{5}$ The first two components $\mathcal{A}$ and $\llbracket-\rrbracket$ of the abstract interpretation are designed so as to ensure that all the post fixpoints of this function $F$ correctly approximate concrete program invariants. The next step of the abstract interpretation is to compute a post fixpoint of $F$ (i.e., some $f$ with $F(f) \sqsubseteq f$ ) using the postfixpoint-computation strategy. This post fixpoint becomes the result of the abstract interpretation.

Throughout the article, we will use two abstract interpretations to explain the main ideas. The first one, evenness analysis, will be mainly used to help the reader to understand the ideas themselves. The second, zone analysis, will be used to illustrate the significance and subtlety of the ideas.

Example 2.1 (Evenness Analysis). The goal of evenness analysis is to discover (at each program point) the variables that always store even numbers. Let EV be a poset $\left\{\perp_{e}\right.$, even, $\left.T_{e}\right\}$ ordered by

$$
\perp_{e} \sqsubseteq_{e} \text { even } \sqsubseteq_{e} T_{e} .
$$

Each element in EV means a set of integers: $\perp_{e}$ denotes the empty set, even the set of all even integers, and $T_{e}$ the set of all integers. Note that the poset EV is a join semilattice; the least element is $\perp_{e}$ and the join operation $\sqcup_{e}$ picks the bigger element among its arguments. The abstract domain $\mathcal{P}=(P, \perp, \sqsubseteq)$ of evenness analysis is given below:

$$
\begin{aligned}
& P \stackrel{\text { def }}{=}[\text { Vars } \rightarrow \mathrm{EV}] \quad a \sqsubseteq a^{\prime} \stackrel{\text { def }}{\Leftrightarrow} \forall x \in \text { Vars. } a(x) \sqsubseteq_{e} a^{\prime}(x) \\
& \perp \stackrel{\text { def }}{=} \lambda x . \perp_{e} \quad a \sqcup a^{\prime} \stackrel{\text { def }}{=} \lambda x . a(x) \sqcup_{e} a^{\prime}(x) .
\end{aligned}
$$

Intuitively, each abstract value $a$ in $P$ specifies which variables should have even numbers. Formally, the meaning of $a$ is given by the following concretization map $\gamma$ from $P$ to States $=$ [Vars $\rightarrow$ Ints]:

$\gamma(a) \stackrel{\text { def }}{=} \begin{cases}\{\sigma \mid \forall x .(a(x)=\text { even } \Rightarrow \sigma(x) \text { is even })\} & \text { if }\left(\forall x . a(x)=\text { even } \vee a(x)=\top_{e}\right) \\ \{\} & \text { otherwise. }\end{cases}$

${ }^{4}$ We use the subscript ${ }_{m}$ to express the monotonicity of functions. Thus, for all posets $(C$, 5$)$ and $\left(C^{\prime}, \complement^{\prime}\right), C \rightarrow_{m} C^{\prime}$ is the poset of monotone functions from $C$ to $C^{\prime}$.

${ }^{5}$ For all $f, g$ in $\prod_{n \in V} \mathcal{A}, f \sqsubseteq g$ iff $\forall n \in V . f(n) \sqsubseteq g(n)$. The least element $\perp$ and join $f \sqcup g$ in this join semilattice are, respectively, defined by $\lambda n . \perp$ and $\lambda n . f(n) \sqcup g(n)$. 
For the abstract semantics of each atomic term, the analysis uses the following definition:

$$
\begin{aligned}
\llbracket x:=2 E \rrbracket a & \stackrel{\text { def }}{=} a[x \mapsto \text { even }] \\
\llbracket x:=y \rrbracket a & \stackrel{\text { def }}{=} a[x \mapsto a(y)] \\
\llbracket x:=E \rrbracket a & \stackrel{\text { def }}{=} a\left[x \mapsto \top_{e}\right] \quad \text { (for all the other assignments) } \\
\llbracket \text { skip } \rrbracket a & \stackrel{\text { def }}{=} a \\
\llbracket E \leq E^{\prime} \rrbracket a & \stackrel{\text { def }}{=} a .
\end{aligned}
$$

In addition, we consider a special atomic term even? $(x)$ only for evenness analysis, which tests whether variable $x$ is even. Its abstract semantics is defined as follows:

$$
\llbracket \text { even? }(x) \rrbracket a \stackrel{\text { def }}{=} \text { if }\left(\text { even } \sqsubseteq_{e} a(x)\right) \text { then } a[x \mapsto \text { even }] \text { else } a
$$

Note that in the semantics, the information "evenness" is created by $x:=2 E$, propagated by $x:=y$, and removed by all the other assignments. Thus, when the analysis is given (the control flow graph of) the code " $\mathrm{x}:=2 \mathrm{x} ; \mathrm{y}:=\mathrm{x} ; \mathrm{x}:=1$," it returns the following annotation for the code:

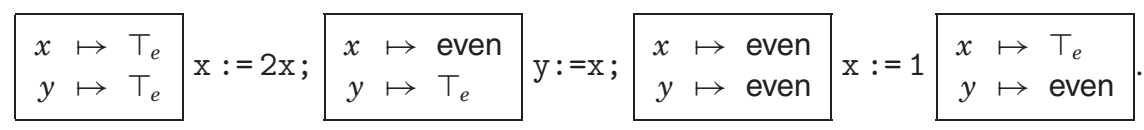

Example 2.2 (Zone Analysis). Zone analysis [Miné 2001] estimates the upper and lower bounds on the difference $x-y$ between two program variables, using so-called difference-bound matrices (in short, DBMs). In this article, we will use a simplified version of zone analysis to illustrate our technique for the relational abstract interpretations. Although we use a simplified version, all the definitions and algorithms in this example are essentially the ones by Miné [2001]. Let $N$ be the number of the program variables in a given program, and let $x_{1}, \ldots, x_{N}$ be an enumeration of all those variables. A DBM $a$ for this program is an $(N+1) \times(N+1)$ matrix with integer values, $-\infty$ or $\infty$. Intuitively, each $a_{i j}$ entry denotes the upper bound of $x_{j}-x_{i}$ (that is, $x_{j}-x_{i} \leq a_{i j}$ ). The row and column of a DBM include an entry for an "auxiliary variable" $x_{0}$ that never appears in the program, and is assumed to have a fixed value 0 . The main role of $x_{0}$ is to allow each DBM to express the range of all the other program variables. For instance, a DBM $a$ can store $l$ in the $i 0$-th entry (i.e., $a_{i 0}=l$ ) for each $i \neq 0$, to record that $-l \leq x_{i}$. A DBM $a$ means the conjunction of $x_{0}=0$ and all the constraints $x_{j}-x_{i} \leq a_{i j}$. Formally, the abstract domain is defined by the following join semilattice $\mathcal{M}=(M, \sqsubseteq, \perp, \sqcup)$ of the DBMs:

$$
\begin{array}{cr}
M & \stackrel{\text { def }}{=}\{a \mid a \text { is a DBM }\} \\
\perp_{i j} \stackrel{\text { def }}{=}-\infty & a \sqsubseteq a^{\prime} \stackrel{\text { def }}{=} \forall i j . a_{i j} \leq a_{i j}^{\prime} \\
{\left[a \sqcup a^{\prime}\right]_{i j} \stackrel{\text { def }}{=} \max \left(a_{i j}, a_{i j}^{\prime}\right) .}
\end{array}
$$




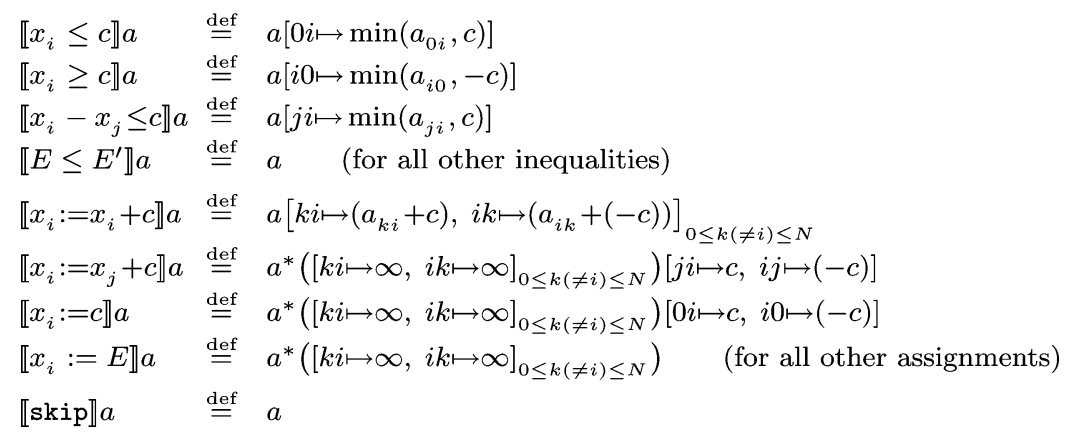

Fig. 3. Abstract semantics of atomic terms in zone analysis.

The formal meaning of each DBM is given by a concretization map (i.e., meaning function) $\gamma$ from $M$ to the powerset of states:

$$
\begin{aligned}
& \text { States } \stackrel{\text { def }}{=}\left[\left\{x_{1}, \ldots, x_{N}\right\} \rightarrow \text { Ints }\right] \\
& \gamma(a) \stackrel{\text { def }}{=}\left\{\sigma \in \text { States } \mid \forall i j . \sigma\left[x_{0} \mapsto 0\right]\left(x_{j}\right)-\sigma\left[x_{0} \mapsto 0\right]\left(x_{i}\right) \leq a_{i j}\right\},
\end{aligned}
$$

where $\sigma\left[x_{0} \mapsto 0\right]$ means the extension of state $\sigma$ with an additional component for $x_{0}: \sigma\left[x_{0} \mapsto 0\right]\left(x_{i}\right) \stackrel{\text { def }}{=}$ if $(i=0)$ then 0 else $\sigma\left(x_{i}\right)$. For instance, $a_{0}$ in Figure 2(b) means the conjunction of five constraints for variables $x_{1}$ and $x_{2}$; these constraints say that $x_{1}$ and $x_{2}$ are, respectively, in the intervals [1, 4] and [1,3], and that $x_{1}$ is at most as big as $x_{2}$. Note that all the diagonal entries of $a_{0}$ are $\infty$, while those entries, meaning the upper bounds for $x_{i}-x_{i}$, could be tighter bound 0 . In this article, we decide to use $\infty$, rather than 0 , for diagonal entries of DBMs, because both $\infty$ and 0 at the diagonal positions provide no information about concrete states and this is clarified by $\infty$.

The analysis classifies atomic terms into two groups, and defines the abstract semantics of the terms in each group in a different style. The first group includes atomic terms whose execution can be precisely modelled by DBM transformations. It consists of inequalities of the form $x_{i} \leq c, x_{i} \geq c, x_{i}-x_{j} \leq c$, assignments of the form $x_{i}:=x_{i}+c, x_{i}:=x_{j}+c, x_{i}:=c$, and command skip. For each inequality $E \leq E^{\prime}$ in the group, $\llbracket E \leq E^{\prime} \rrbracket$ calculates the conjunction of $E \leq E^{\prime}$ and the constraints denoted by the input DBM; for each assignment $x:=E$ in this group, $\llbracket x:=E \rrbracket$ computes its strongest postcondition for the input DBM; and 【skip》 is defined to be the identity function. The semantics of these atomic terms is shown in Figure 3. The abstract semantics of $x_{i}-x_{j} \leq c$ in Figure 3 implements the pruning of states, by updating the $j i$-th entry of the input DBM $a$ by $\min \left(a_{j i}, c\right)$. Note that the updated DBM means precisely the conjunction of $x_{i}-x_{j} \leq c$ and $\gamma(a)$. Thus, among the states in (the concretization of) the input DBM, $\llbracket x_{i}-x_{j} \leq c \rrbracket$ filters out the states that violate the condition $x_{i}-x_{j} \leq c$, and returns a DBM for the remaining states. The abstract semantics $\llbracket x_{i}:=x_{i}+c \rrbracket$ models the increment of $x_{i}$ by $c$. For every $k l$-th entry of $a$, if the column index $l$ is $i$, so the entry means the constraint involving $x_{i}, \llbracket x_{i}:=x_{i}+c \rrbracket$ increments the entry by $c$; and if the row index $k$ is $i$, so the entry now means the constraint involving $-x_{i}$, not $x_{i}, \llbracket x_{i}:=x_{i}+c \rrbracket$ decrements the entry by $c$. The case $\llbracket x_{i}:=x_{j}+c \rrbracket$ in the figure is the most complex and interesting. Given 
a DBM $a$, the semantic function $\llbracket x_{i}:=x_{j}+c \rrbracket$ first transforms $a$, so that the DBM has the smallest element $a^{*}$ among the ones that mean the same state set as $a$ : $a^{*}$ satisfies $\gamma(a)=\gamma\left(a^{*}\right)$, and for all other such DBMs $a^{\prime}\left(\right.$ i.e., $\gamma\left(a^{\prime}\right)=\gamma(a)$ ), $a^{*} \sqsubseteq a^{\prime}$. We call $a^{*}$ the closure of $a$. Zone analysis computes this closure using the Floyd-Warshall shortest path algorithm. ${ }^{6}$ Next, $\llbracket x_{i}:=x_{j}+c \rrbracket$ eliminates all the information in $a^{*}$ involving the old value of $x_{i}$. Finally, it adds two facts, $x_{i}-x_{j} \leq c$ and $x_{j}-x_{i} \leq-c$.

The atomic terms in the other group are interpreted "syntactically": the semantics of an assignment $x_{i}:=E$ in this group does not consider the expression $E$, and transforms an input DBM $a$ to the following $x_{i}$-deleted DBM:

$$
a^{*}\left([k i \mapsto \infty, i k \mapsto \infty]_{0 \leq k(\neq i) \leq N}\right),
$$

and the semantics of $E \leq E^{\prime}$ in the group prunes nothing, and means the identity function on the DBMs. A better alternative is to use interval analysis to give the semantics of atomic terms in the second group as shown by Miné [2001]. In Section 5, we will discuss this better semantics and other improvements used in the original zone analysis [Miné 2001].

Figure 2(c) shows a result of zone analysis in the form of DBMs and constraints. The input to zone analysis is the program in Figure 2(a) and the DBM $a_{0}$ in Figure 2(b). The result implies that when the program terminates, $x_{2}$ is in the interval $[1,3]$ and it is equal to $x_{1}-1$.

\section{ABSTRACT-VALUE SLICER}

An abstract-value slicer is an algorithm that filters out unnecessary information from the result of an abstract interpretation. When an abstract interpretation is used for verification, it usually computes stronger invariants than needed. This situation commonly happens, because an abstract interpretation is usually designed and implemented to blindly estimate best possible invariants at each program point without considering global goal of the intended verification, normally assuming that every aspect of a program potentially contributes to the properties of interest. However, in the verification of a specific safety property, only some aspects of the program are usually needed. As a result, the abstract interpretation results are likely to contain unnecessary information for such verification. Actually, this situation results from a design choice too, because we want one abstract interpretation to estimate invariants once for multiple verifications of different safety properties.

The goal of an abstract-value slicer is to weaken the computed invariants until no information in the invariants is unnecessary for a specific verification. Mathematically, the abstract-value slicer lifts the result $f$ of an abstract interpretation: it computes a new post fixpoint $f^{\prime}$ of the abstract step function $F$ in Section 2, such that $f \sqsubseteq f^{\prime}$, but $f^{\prime}$ is still strong enough to prove the properties of interest. Intuitively, the "difference" between $f$ and $f^{\prime}$ represents the information filtered out from $f$ by the abstract-value slicer.

${ }^{6}$ When the shortest path algorithm is applied, program variables are considered nodes in the graph and each DBM entry $a_{j i}$ is regarded as the weight of the edge from node $x_{j}$ to node $x_{i}$. 
In this section, we define the abstract-value slicers, and prove their correctness. First, we introduce extractor domain and back-tracers for atomic terms, which are two main components of an abstract-value slicer. An extractor domain determines the working space of an abstract-value slicer, that is, a poset where the abstract-value slicer does the fixpoint computation, and back-tracers specify how the abstract-value slicer treats atomic terms: they describe how the slicer filters out unnecessary information from the abstract interpretation results for atomic terms. Then, we define an abstract-value slicer, and prove its correctness. Throughout the section, we assume a fixed abstract interpretation, and denote its abstract domain and abstract semantics of atomic terms by $\mathcal{A}=(A, \sqsubseteq, \perp, \sqcup)$ and $\llbracket-\rrbracket$, respectively.

\subsection{Extractor Domain}

An extractor domain for the abstract interpretation $(\mathcal{A}, \llbracket-\rrbracket)$ is a pair $(\mathcal{E}$, ex) where $\mathcal{E}$ is a complete lattice $\left(\complement_{\mathcal{E}}, \perp_{\mathcal{E}}, \top_{\mathcal{E}}, \sqcup_{\mathcal{E}}, \Pi_{\mathcal{E}}\right)$ with finite height ${ }^{7}$ and ex is a monotone map from $\mathcal{E}$ to upper closure operators on $\mathcal{A} .^{8}$ Intuitively, each element $e$ in $\mathcal{E}$ denotes an "information extractor" that selects some information from abstract values $a$ in $\mathcal{A}$, which is to be saved/preserved, and ex $(e)(a)$ extracts information (to be saved/preserved) from $a$ based on the extractor $e$. Note that we require ex(e) to be an upper closure operator, that is, a monotone function that satisfies extensiveness and idempotency requirements. The extensiveness requirement means that the extracting operation $\operatorname{ex}(e)(a)$ lifts the value $a$. When an extractor $e$ is applied to the abstract value $a$, it does not insert any new information, but only selects some information from $a$; thus, ex $(e)(a)$ should have less information than $a$ (i.e., $a \sqsubseteq \operatorname{ex}(e)(a)$ ). The idempotency requirement formalizes that the extraction by ex(e) is done all at once. We also point out that ex should be monotone with respect to the order $\Xi_{\mathcal{E}}$ on extractors. This monotonicity condition ensures that the order $\sqsubseteq_{\mathcal{E}}$ means the "strength" of the information extractors in the reverse direction: if $e \sqsubseteq_{\mathcal{E}} e^{\prime}$, then $e^{\prime}$ extracts less information than $e$.

We call $\mathcal{E}$ extractor lattice and ex extractor application. We often omit the subscript $-_{\mathcal{E}}$ in the lattice operators, $\sqsubseteq_{\mathcal{E}}, \perp_{\mathcal{E}}, \top_{\mathcal{E}}, \sqcup_{\mathcal{E}}, \Pi_{\mathcal{E}}$, when the missing subscript can be recovered from context.

Example 3.1. We use the following extractor domain for evenness analysis: $\mathcal{E} \stackrel{\text { def }}{=} \wp($ Vars $)$ (ordered by $\supseteq)$ and $\operatorname{ex}(e)(a) \stackrel{\text { def }}{=} \lambda x$. if $x \in e$ then $a(x)$ else $T_{e}$.

In this extractor domain, each abstract value $a$ is regarded as the conjunction of information " $x \mapsto a(x)$ " for all $x \in$ Vars, and the extractors in $\mathcal{E}$ indicate which information should be selected from such conjunction. For instance, an abstract value $[x \mapsto$ even, $y \mapsto$ even, $z \mapsto$ even] is regarded as even $(x) \wedge$ even $(y) \wedge$ even $(z)$, where the predicate even $(x)$ asserts that $x$ is even, and the extractor $\{x, y\}$ expresses that only the first and second conjuncts, even $(x) \wedge$ even $(y)$, should be

\footnotetext{
${ }^{7}$ We don't need to require the completeness of $\mathcal{E}$, since all lattices with finite height are complete. However, we put the completeness requirement explicitly here to simplify presentation.

${ }^{8}$ An upper closure operator $\rho$ on $\mathcal{A}$ is a monotone function on $\mathcal{A}$ such that $\rho$ is extensive (i.e., id $\sqsubseteq \rho$ ) and idempotent (i.e., $\rho \circ \rho=\rho$ ).
} 
selected. Note that the definition formalizes such selection using the top element $T_{e}$ of EV: all the unselected information is replaced by $T_{e}$, while the other selected information remains as it is. The extractor ex $(e)$ for evenness analysis is an upper closure operator: it is extensive because $\forall a \in \mathcal{A}$. $\forall x \in \operatorname{Vars.} a(x) \sqsubseteq$ $\operatorname{ex}(e)(a)(x)$, and idempotent because $\forall a \in \mathcal{A}$. $\operatorname{ex}(e)(\operatorname{ex}(e)(a))=\operatorname{ex}(e)(a)$.

Example 3.2. We construct an extractor domain $(\mathcal{E}$, ex) for zone analysis, using a set of matrix indices as an information extractor. The idea is to use each index set $e$ to specify which entries of the DBM matrices should be extracted. For each DBM matrix $a$, ex $(e)(a)$ selects only the entries of $a$ whose indices are in $e$, and it fills in the other missing entries by $\infty$. For example, when the extractor $\{(2,1)\}$ is applied to the DBM $a_{0}$ in Figure 2(b), it filters out all entries except the $(2,1)$-th entry, and results in the below DBM, which means $x_{1}-x_{2} \leq 0$.

$$
\begin{array}{l|l} 
& x_{0} x_{1} x_{2} \\
\hline x_{0} & \infty \infty \infty \\
x_{1} & \infty \infty \infty \\
x_{2} & \infty 0 \infty
\end{array}
$$

Let $N$ be the number of program variables, so that the domain $\mathcal{M}$ of DBMs consists of $(N+1) \times(N+1)$ matrices, and let $I$ be the index set $(N+1) \times(N+1)$. The precise definition of $\mathcal{E}$ and ex is given below:

$$
\mathcal{E} \stackrel{\text { def }}{=}\langle\wp(I), \supseteq, I, \emptyset, \cap, \cup\rangle \text { and }(\operatorname{ex}(e)(a))_{i j} \stackrel{\text { def }}{=} \begin{cases}a_{i j} & \text { if } i j \in e \\ \infty & \text { otherwise }\end{cases}
$$

Note that the extractor lattice uses the superset order; thus, a smaller extractor selects more matrix entries from the input DBM than a bigger one.

\subsection{Back-Tracers}

Let $(\mathcal{E}$, ex) be an extractor domain for the abstract interpretation $(\mathcal{A}, \mathbb{\llbracket}-\mathbb{\rrbracket})$. For each atomic term $t$, define prepost $(t)$ by

$$
\operatorname{prepost}(t) \stackrel{\text { def }}{=}\{(a, b) \mid a, b \in \mathcal{A} \wedge \llbracket t \rrbracket a \sqsubseteq b\},
$$

which means the pre and post conditions of the triples $\{a\} t\{b\}$ for $t$ that can be proved by the abstract interpretation.

Definition 3.3 (Back-Tracer). A back-tracer $k$ for an atomic term $t$ is a function of type prepost $(t) \rightarrow \mathcal{E} \rightarrow \mathcal{E}$ that satisfies the following soundness condition:

$$
\forall(a, b) \in \operatorname{prepost}(t) . \forall e, e^{\prime} \in \mathcal{E} .\left(k_{a b}(e)=e^{\prime}\right) \Longrightarrow \llbracket t \rrbracket\left(\operatorname{ex}\left(e^{\prime}\right)(a)\right) \sqsubseteq \operatorname{ex}(e)(b) .
$$

The back-tracer $k_{a b}$ at $(a, b)$ transforms a post extractor $e$ (for $b$ ) to a pre extractor $e^{\prime}$ (for $a$ ). The soundness condition ensures that the $e^{\prime}$-part of $a$ is sufficient to get the $e$-part of $b$ in the abstract interpretation.

A back-tracer induces a map from $\{\operatorname{ex}(e)(b) \mid e \in \mathcal{E}\}$ to $\{\operatorname{ex}(e)(a) \mid e \in \mathcal{E}\}$, when it satisfies

$$
\operatorname{ex}(e)(b)=\operatorname{ex}\left(e^{\prime}\right)(b) \Longrightarrow \operatorname{ex}\left(k_{a b}(e)\right)(a)=\operatorname{ex}\left(k_{a b}\left(e^{\prime}\right)\right)(a) .
$$


The domain and codomain of this map represent pieces of information from $b$ and $a$, respectively, so the map indicates that the back-tracer transforms a piece of information of $b$ to another piece of information of $a$.

Note that the back-tracer $k_{a b}$ for an atomic term $t$ is not required to be monotone. Thus, it is relatively easy to design one correct back-tracer. For example, suppose that $\operatorname{ex}(\perp)$ is the identity function. ${ }^{9}$ Then, for every $e \in \mathcal{E}$, there exists $e^{\prime} \in \mathcal{E}$ such that

$$
\llbracket t \rrbracket\left(\operatorname{ex}\left(e^{\prime}\right)(a)\right) \sqsubseteq \operatorname{ex}(e)(b),
$$

because $\perp$ could be $e^{\prime}$. We can now define $k_{a b}(e)$ to be one such $e^{\prime}$. However, designing a good back-tracer, not just correct one, is a nontrivial problem, and requires insights about the abstract interpreter and the extractor domain.

Our next result, Proposition 3.6, is concerned with this issue of designing good back-tracers. It gives a sufficient and necessary condition for the existence of the best back-tracers. In Section 4, we will discuss this issue further, and provide general techniques for implementing good back-tracers, including the best ones.

Definition 3.4 (Best Back-Tracer). A back-tracer $k$ for an atomic term $t$ is best if and only if it is the greatest back-tracer for $t:{ }^{10}$ for all back-tracers $k^{\prime}$ for $t$,

$$
\forall(a, b) \in \operatorname{prepost}(t) . \forall e \in \mathcal{E} . \quad k_{a b}^{\prime}(e) \sqsubseteq k_{a b}(e) .
$$

Lemma 3.5. A function $k: \operatorname{prepost}(t) \rightarrow \mathcal{E} \rightarrow \mathcal{E}$ is the best back-tracer for $t$ if and only if for all $(a, b) \in \operatorname{prepost}(t)$ and all $e, e^{\prime} \in \mathcal{E},{ }^{11}$

$$
e^{\prime} \sqsubseteq k_{a b}(e) \Longleftrightarrow \llbracket t \rrbracket\left(\operatorname{ex}\left(e^{\prime}\right)(a)\right) \sqsubseteq \operatorname{ex}(e)(b) .
$$

Proof. First, we prove the only-if direction. Suppose that $k$ is the best backtracer for $t$, and choose arbitrary $(a, b) \in \operatorname{prepost}(t)$ and $e, e^{\prime} \in \mathcal{E}$. We need to show the equivalence:

$$
e^{\prime} \sqsubseteq k_{a b}(e) \Longleftrightarrow \llbracket t \rrbracket\left(\operatorname{ex}\left(e^{\prime}\right)(a)\right) \sqsubseteq \operatorname{ex}(e)(b) .
$$

Suppose that the left-hand side of the equivalence holds. Then,

$$
\begin{array}{rlrl}
\llbracket t \rrbracket\left(\operatorname{ex}\left(e^{\prime}\right)(a)\right) & \left.\sqsubseteq \llbracket t \rrbracket\left(\operatorname{ex}\left(k_{a b}(e)\right)(a)\right) \quad \text { (by the monotonicity of } \llbracket t \rrbracket \text { and ex }(-)(a)\right) \\
& \sqsubseteq \operatorname{ex}(e)(b) & \text { (by the soundness of the back-tracer } k) .
\end{array}
$$

Thus, the right-hand side of the equivalence holds as well. Now suppose that the right-hand side of the equivalence holds. Define a function $k^{\prime}$ as follows:

$$
\begin{aligned}
k^{\prime} & : \operatorname{prepost}(t) \rightarrow \mathcal{E} \rightarrow \mathcal{E} \\
k_{c d}^{\prime}\left(e_{0}\right) \stackrel{\text { def }}{=} & \text { if }\left(\left(c, d, e_{0}\right)=(a, b, e)\right) \text { then } e^{\prime} \text { else } k_{c d}\left(e_{0}\right)
\end{aligned}
$$

\footnotetext{
${ }^{9}$ This supposition holds for all the examples in this paper.

${ }^{10}$ This definition can be rephrased as follows. A function $k$ is the best back-tracer for $t$ if and only if $k$ is the least upper bound of all back-tracers for $t$ and $k$ itself is a back-tracer for $t$.

${ }^{11}$ The equivalence between the order relationships is reminiscent of Galois connection. We cannot use Galois connection directly here, because $k_{a b}: \mathcal{E} \rightarrow \mathcal{E}$ and $\llbracket t \rrbracket: \mathcal{A} \rightarrow \mathcal{A}$ are not type-correct for Galois connection. We resolve this type error using functions ex $(-)(a)$ ex $(-)(b)$ of type $\mathcal{E} \rightarrow \mathcal{A}$.
} 
$k^{\prime}$ is a back-tracer because it satisfies the condition for back-tracers: when the arguments are $(a, b, e), k_{a b}^{\prime}(e)=e^{\prime}$ holds and the right-hand side of the equivalence directly means the condition for back-tracers, and when the arguments are not $(a, b, e), k^{\prime}$ is the same as $k$, which is already a back-tracer. Since $k$ is a best back-tracer, $k^{\prime} \sqsubseteq k$ holds, which implies the left-hand side of the equivalence as follows:

$$
e^{\prime}=k_{a b}^{\prime}(e) \sqsubseteq k_{a b}(e) .
$$

Next, we prove the if direction. Suppose that $k$ satisfies the equivalence in the lemma. Then, for all back-tracers $k^{\prime}$ for $t$, and all $(a, b) \in \operatorname{prepost}(t)$ and $e \in \mathcal{E}$

$$
\llbracket t \rrbracket\left(\operatorname{ex}\left(k_{a b}^{\prime}(e)\right)(a)\right) \sqsubseteq \operatorname{ex}(e)(b) \quad \text { by the soundness of back-tracer } k^{\prime},
$$

and so, by the equivalence in the lemma, $k_{a b}^{\prime}(e) \sqsubseteq k_{a b}(e)$. We just have shown that $k^{\prime}$ is smaller than or equal to $k$. It remains to show that $k$ is a back-tracer for $t$, that is, it satisfies the soundness requirement for back-tracers for $t$. To show the requirement, consider arbitrary $(a, b) \in \operatorname{prepost}(t)$ and $e \in \mathcal{E}$. Let $e^{\prime}$ be $k_{a b}(e)$. Since $e^{\prime} \sqsubseteq k_{a b}(e)$, the equivalence in the lemma gives

$$
\llbracket t \rrbracket\left(\operatorname{ex}\left(e^{\prime}\right)(a)\right) \sqsubseteq \operatorname{ex}(e)(b) .
$$

Note that this order relationship is precisely the soundness requirement for back-tracers for $t$.

Proposition 3.6. An atomic term $t$ has the best back-tracer if for all $a \in \mathcal{A}$, the function $\lambda e . \llbracket t \rrbracket(\operatorname{ex}(e)(a)): \mathcal{E} \rightarrow_{m} \mathcal{A}$ preserves all finite joins. Moreover, when $\operatorname{ex}(\perp)$ is the identity function on $\mathcal{A}$, the converse holds as well.

Proof. First, we prove the if direction. Suppose that for all $a \in \mathcal{A}$, function $\lambda e . \llbracket t \rrbracket(\operatorname{ex}(e)(a))$ of type $\mathcal{E} \rightarrow_{m} \mathcal{A}$ preserves all finite joins. Define a function $k$ as follows:

$$
\begin{gathered}
k: \operatorname{prepost}(t) \rightarrow \mathcal{E} \rightarrow \mathcal{E} \\
k_{a b}(e) \stackrel{\text { def }}{=} \bigsqcup\left\{e_{0} \mid \llbracket t \rrbracket\left(\operatorname{ex}\left(e_{0}\right)(a)\right) \sqsubseteq \operatorname{ex}(e)(b)\right\} .
\end{gathered}
$$

We will show that $k$ satisfies the following equivalence in Lemma 3.5: for all $e, e^{\prime} \in \mathcal{E}$,

$$
e^{\prime} \sqsubseteq k_{a b}(e) \Longleftrightarrow \llbracket t \rrbracket\left(\operatorname{ex}\left(e^{\prime}\right)(a)\right) \sqsubseteq \operatorname{ex}(e)(b) .
$$

The right-to-left implication follows from the definition of $k$. For the left-to-right implication, it is sufficient (because $\lambda e . \llbracket t \rrbracket(\operatorname{ex}(e)(a))$ is monotone) to prove the following condition:

$$
e^{\prime}=k_{a b}(e) \Longrightarrow \llbracket t \rrbracket\left(\operatorname{ex}\left(e^{\prime}\right)(a)\right) \sqsubseteq \operatorname{ex}(e)(b) .
$$

Suppose that $e^{\prime}=k_{a b}(e)$. Since the extractor domain has finite height, there exists a finite nonempty subset $E_{0}$ of $\left\{e_{0} \mid \llbracket t \rrbracket\left(\operatorname{ex}\left(e_{0}\right)(a)\right) \sqsubseteq \operatorname{ex}(e)(b)\right\}$ such that $e^{\prime}=$ $\sqcup E_{0}$. Note that by the assumption of the if direction, function $\lambda e$. $\llbracket t \rrbracket(\operatorname{ex}(e)(a))$ preserves finite joins. From this join preservation and the choice of $E_{0}$, we 
derived the required implication:

$$
\begin{aligned}
\llbracket t \rrbracket\left(\operatorname{ex}\left(e^{\prime}\right)(a)\right) & =\llbracket t \rrbracket\left(\operatorname{ex}\left(\bigsqcup E_{0}\right)(a)\right) & & \left(\text { since } e^{\prime}=\bigsqcup E_{0}\right) \\
& =\bigsqcup_{e_{0} \in E_{0}} \llbracket t \rrbracket\left(\operatorname{ex}\left(e_{0}\right)(a)\right) & & \text { (by the join preservation of } \llbracket t \rrbracket(\operatorname{ex}(-)(a))) \\
& \sqsubseteq \operatorname{ex}(e)(b) & & \left(\text { since } \forall e_{0} \in E_{0} . \llbracket t \rrbracket\left(\operatorname{ex}\left(e_{0}\right)(a)\right) \sqsubseteq \operatorname{ex}(e)(b)\right) .
\end{aligned}
$$

Next, we show the only if direction, assuming that ex $(\perp)$ is the identity on $\mathcal{A}$. Suppose that $t$ has the best back-tracer $k$. Then, it satisfies the equivalence in Lemma 3.5. Consider a finite family $\left\{e_{i}\right\}_{i \in I}$ of extractors. Then, by the monotonicity of ex and $\llbracket t \rrbracket$, we have that

$$
\llbracket t \rrbracket\left(\operatorname{ex}\left(\bigsqcup_{i \in I} e_{i}\right)(a)\right) \sqsupseteq \bigsqcup_{i \in I} \llbracket t \rrbracket\left(\operatorname{ex}\left(e_{i}\right)(a)\right) .
$$

Thus, to show that the join in $\bigsqcup_{i \in I} e_{i}$ is preserved, we only need to prove the other order relationship. Let $b$ be $\bigsqcup_{i \in I} \llbracket t \rrbracket\left(\operatorname{ex}\left(e_{i}\right)(a)\right)$. Then,

$$
\begin{array}{llr} 
& \forall i \in I . \llbracket t \rrbracket\left(\operatorname{ex}\left(e_{i}\right)(a)\right) \sqsubseteq b & \text { (by the definition of } b) \\
& \forall i \in I . \llbracket t \rrbracket\left(\operatorname{ex}\left(e_{i}\right)(a)\right) \sqsubseteq \operatorname{ex}(\perp)(b) & \text { (since ex }(\perp) \text { is the identity) } \\
\Longleftrightarrow \quad & \forall i \in I . e_{i} \sqsubseteq k_{a b}(\perp) & \text { (by the equivalence in Lemma 3.5) } \\
& \left(\bigsqcup_{i \in I} e_{i}\right) \sqsubseteq k_{a b}(\perp) & \\
& \llbracket t \rrbracket\left(\operatorname{ex}\left(\bigsqcup_{i \in I} e_{i}\right)(a)\right) \sqsubseteq \operatorname{ex}(\perp)(b) \quad \text { (by the equivalence in Lemma 3.5) } \\
& \llbracket t \rrbracket\left(\operatorname{ex}\left(\bigsqcup_{i \in I} e_{i}\right)(a)\right) \sqsubseteq b
\end{array}
$$

We have just shown the required order relationship.

Example 3.7. We define a back-tracer for each atomic term for evenness analysis. Recall the abstract domain $\mathcal{P}$ of evenness analysis in Example 2.1, and the extractor domain $(\mathcal{E}$, ex) for the analysis in Example 3.1. For a back-tracer for an atomic term $t$, we use $(|t|)$ for notational convenience. The back-tracer $(|t|)$ for each atomic term $t$ in this domain is defined as follows:

$$
\begin{aligned}
\left(\left.|x:=2 E|\right|_{a b}(e)\right. & \stackrel{\text { def }}{=} e-\{x\}, \\
\left(\left.|x:=y|\right|_{a b}(e)\right. & \stackrel{\text { def }}{=} \text { if } x \in e \text { then }(e-\{x\}) \cup\{y\} \text { else } e, \\
(|x:=E|)_{a b}(e) & \stackrel{\text { def }}{=} e-\{x\} \quad \text { for all the other assignments), } \\
\quad\left(\mid \text { skip }\left.\right|_{a b}(e)\right. & \stackrel{\text { def }}{=} e \\
\left(\left|E \leq E^{\prime}\right|\right)_{a b}(e) & \stackrel{\text { def }}{=} e \\
(\text { even? } & =
\end{aligned}
$$

The back-tracer for every assignment $x:=E$ has the same pattern. Given an extractor $e$, it first deletes $x$ from $e$, and then adds (to the resulting extractor) the variables used in the abstract semantics. Note that this is similar to the DEFUSE calculation in the conventional data-flow analysis. The main difference is that the back-tracer computes the DEF-USE for the abstract semantics, not 
for the concrete semantics. For instance, when the back-tracer $\left(|\mathrm{x}:=\mathrm{y}+\mathrm{z}|_{a b}\right.$ is applied to the extractor $\{x, v\}$, it deletes $x$ from the extractor and returns $\{v\}$. The variables $y, z$ are not added to the extractor even though they are read by assignments in the concrete semantics. This is because $y, z$ are not used by the abstract semantics of the assignments. The back-tracer for evenness test even? $(x)$ uses the analysis results critically. The function \even? $(x) \rrbracket$ refines the input $a$ by replacing $a(x)$ by the minimum of $a(x)$ and even. Thus, for pre and post conditions $(a, b) \in$ prepost(even? $(x))$ (i.e., \even? $(x) \rrbracket a \sqsubseteq b)$, if even $\sqsubseteq_{e} b(x)$, then the $x$ component of $a$ is not necessary to obtain the $x$ component of $b$. The back-tracer (even? $\left.(x)\right|_{a b}$ correctly captures this using the analysis result $b$; it first tests whether even $\sqsubseteq_{e} b(x)$, and if so, it deletes $x$ from the given extractor $e$.

Example 3.8. Recall the abstract domain $\mathcal{M}$ for zone analysis in Example 2.2 and the extractor domain $(\mathcal{E}$, ex) for the analysis in Example 3.2. The back-tracer $(|t|)$ for an atomic term $t$ in this extractor domain should be a parameterized index-set transformer that satisfies the following condition: for all pre and post conditions $(a, b) \in \operatorname{prepost}(t)$ (i.e., $\llbracket t \rrbracket a \sqsubseteq b$ ) and all extractors $e$ for $b$, the computed index set $(|t|)_{a b}(e)$ contains (the indices of) all the entries of $a$ that are necessary for obtaining the $e$ entries of $b$. We define such a back-tracer $(|t|)$ as a two-step computation. First, $(t)_{a b}(e)$ deletes all the indices $i j$ from $e$ that satisfy $b_{i j}=\infty$ (i.e., $e-\left\{i j \mid b_{i j}=\infty\right\}$ ). Then, for each remaining $i j$-th entry of $e$, $(\mid t)_{a b}(e)$ computes the entries of $a$ that are needed for obtaining $(\llbracket t \rrbracket a)_{i j}$, collects all the computed entries, and returns the set of the collected indices. Note that all the deleted indices $i j$ in the first step select only the empty information from $b$ : for all index sets $e$, we have that $\operatorname{ex}_{b}(e)=\operatorname{ex}_{b}(e-\{i j\})$. Thus, the first step only makes $e$ have a better representation $e^{\prime}$ (i.e., $e^{\prime} \subseteq e$ ), without changing its effect on $b$. Another thing to note is that the second step is concerned with only $a$ and $\llbracket t \rrbracket a$, but not $b$. Here the second step exploits the fact that to get the $e^{\prime}$ entries of $b$, we need only the $e^{\prime}$ entries of $\llbracket t \rrbracket a$.

The actual implementation $(|t|)$ for each atomic term $t$ optimizes the generic two-step computation, and it is shown in Figure 4. The most interesting part is the last case $x_{i}:=E$. The back-tracer $\left(\left|x_{i}:=E\right|_{a b}(e)\right.$ first checks whether the input matrix $a$ has a negative cycle, that is, a sequence $k_{0} k_{1} \ldots k_{n}$ of integers in $[0, N]$ such that $k_{0}=k_{n}, n \geq 1$, and $\sum_{m=0}^{n-1} a_{k_{m} k_{m+1}}<0$. If $a$ has a negative cycle, $\left(\left|x_{i}:=E\right|_{a b}(e)\right.$ picks a shortest such cycle (i.e., one with smallest $n$ ), and returns the set of all the "edges" $k_{m} k_{m+1}$ in the cycle. If $a$ does not have a negative cycle, $\left(\left|x_{i}:=E\right|_{a b}(e)\right.$ eliminates all the indices $k l$ from $e$ such that $b_{k l}=\infty$ or $i=k \vee i=l$. Then, for each remaining index $k l$ in $e,\left(\left|x_{i}:=E\right|_{a b}(e)\right.$ selects a sequence $k_{0} \ldots k_{n}$ of integers in $[0, N]$ such that $k_{0}=k, k_{n}=l$, and

$$
\left(\sum_{m=0}^{n-1} a_{k_{m} k_{m+1}}\right) \leq(b)_{k l} .
$$

The formula on the left-hand side of the above inequality computes an upper bound of $\left(a^{*}\right)_{k l}$, and the inequality means that this upper bound is still tight enough to prove that $\left(a^{*}\right)_{k l} \leq(b)_{k l}$. The set of all the "edges" $k_{m} k_{m+1}$ in these selected paths is the result of the back-tracer $\left(\left|x_{i}:=E\right|_{a b}(e)\right.$. 


$$
\begin{aligned}
& \left(x_{i} \leq c\right)_{a b}(e) \quad \stackrel{\text { def }}{=} \text { if }\left(b_{0 i} \geq c\right) \text { then }\left(e-\left\{k l \mid b_{k l}=\infty\right\}-\{0 i\}\right) \\
& \text { else }\left(e-\left\{k l \mid b_{k l}=\infty\right\}\right) \\
& \left(x_{i} \geq c\right)_{a b}(e) \quad \stackrel{\text { def }}{=} \text { if }\left(b_{i 0} \geq-c\right) \text { then }\left(e-\left\{k l \mid b_{k l}=\infty\right\}-\{i 0\}\right) \\
& \text { else }\left(e-\left\{k l \mid b_{k l}=\infty\right\}\right) \\
& \left(x_{i}-x_{j} \leq c\right)_{a b}(e) \stackrel{\text { def }}{=} \text { if }\left(b_{j i} \geq c\right) \text { then }\left(e-\left\{k l \mid b_{k l}=\infty\right\}-\{j i\}\right) \\
& \left(E \leq E^{\prime}\right)_{a b}(e) \quad \stackrel{\text { def }}{=} e-\left\{k l \mid b_{k l}=\infty\right\} \\
& \left(x_{i}:=x_{i}+c\right)_{a b}(e) \stackrel{\text { def }}{=} e-\left\{k l \mid b_{k l}=\infty\right\} \\
& \left(x_{i}:=E l_{a b}(e) \quad \stackrel{\text { def }}{=} \text { if (hasNegCycle }(a)=\right.\text { true) } \\
& \text { then edges (pickNegCycle }(a)) \\
& \text { else let } e^{\prime}=\left(e-\left\{k l \mid b_{k l}=\infty\right\}-\{i k, k i \mid 0 \leq k \leq N\}\right) \\
& \text { in } \bigcup_{k l \in e^{\prime}}\left(\text { if } a_{k l} \leq b_{k l} \text { then }\{k l\} \text { else edges }(\operatorname{mPath}(a, k, l))\right) \\
& \text { (where } E \text { is either } x_{j}+c, c \text {, or a general expression } E \text {, and } \\
& (\operatorname{skip})_{a b}(e) \quad \stackrel{\text { def }}{=} e-\left\{k l \mid b_{k l}=\infty\right\} \\
& \text { edges }\left(k_{0} k_{1} \ldots k_{n}\right)=\left\{k_{0} k_{1}, k_{1} k_{2}, \ldots, k_{n-1} k_{n}\right\} \text {. ) }
\end{aligned}
$$

Fig. 4. Back-tracers for atomic terms in zone analysis.

When $\left(\mid x_{i}:=E\right)_{a b}(e)$ chooses a path from $k$ to $l$ in the second step, it usually picks one with the minimum weight, denoted $\mathrm{mPath}(a, k, l) .{ }^{12}$ However, when $a_{k l} \leq b_{k l},\left(\left|x_{i}:=E\right|\right)_{a b}(e)$ selects a possibly different and shorter path $k l$. Note that the selected path for $k l$ here might be different from the path that the abstract interpretation has used to compute the $k l$-th entry of $b$. This shows that $\left(\left|x_{i}:=E\right|\right)_{a b}(e)$ does not necessarily denote the part of $a$ that the abstract interpretation has used to obtain the $e$-part of $b$; instead it means the part of $a$ that the abstract interpretation can use to get the $e$-part of $b$.

To see how the back-tracer works more clearly, consider the following pre and post conditions of $x_{3}:=0:^{13}$

$$
\llbracket x_{3}:=0 \rrbracket\left(\begin{array}{c|cccc} 
& x_{0} & x_{1} & x_{2} & x_{3} \\
\hline x_{0} & \infty & 4 & 3 & \infty \\
x_{1} & -1 & \infty & 4 & \infty \\
x_{2} & -1 & 0 & \infty & \infty \\
x_{3} & \infty & \infty & \infty & \infty
\end{array}\right) \sqsubseteq\left(\begin{array}{c|cccc} 
& x_{0} & x_{1} & x_{2} & x_{3} \\
\hline x_{0} & \infty & 5 & \infty & 0 \\
x_{1} & \infty & \infty & 2 & \infty \\
x_{2} & \infty & \infty & \infty \\
x_{3} & 0 & \infty & \infty & \infty
\end{array}\right) .
$$

Let $a$ and $b$ be, respectively, the left and right DBMs of this relationship. When the back-tracer $\left(\left|x_{3}:=0\right|\right)_{a b}$ is given a post extractor $e=$ $\{(0,1),(1,2),(2,1),(3,0)\}$ it first gets rid of $(2,1)$ and $(3,0)$ from $e$, because the $(2,1)$-th entry of $b$ has $\infty$ and the (3,0)-th entry of $b$ is generated by the assignment $x_{3}:=0$. Then, the back-tracer $\left(\left|x_{3}:=0\right|_{a b}\right.$ computes paths for $(0,1)$

${ }^{12} \mathrm{mPath}(a, k, l)$ is a path $k_{0} \ldots k_{n}$ such that $k_{0}=k, k_{n}=l$ and

$$
\left(\sum_{m=0}^{n-1} a_{k_{m} k_{m+1}}\right)=\left(a^{*}\right)_{k l} \text {. }
$$

${ }^{13}$ The second DBM can arise, for instance, when the assignment $x_{3}:=0$ is executed at the end of the false branch of a conditional statement and the analysis result of the true branch is a DBM whose $(0,2),(1,0),(2,0),(2,1)$ entries are $\infty$ and $(0,1)$ entry is 5 . 
and $(1,2)$ separately; for $(0,1)$, it picks the path 0,1 , because $a_{01} \leq b_{01}$; and for the other index $(1,2)$, condition $a_{12} \leq b_{12}$ does not hold, and so the back-tracer computes a path from 1 to 2 with the minimum weight, which is the sequence $1,0,2$. Finally, it returns the index set $\{(0,1),(1,0),(0,2)\}$ that consists of all the edges in the computed two paths.

Back-tracers for all atomic terms induce a back-tracer for an entire program $P=\left(V, E, n_{i}, n_{f}, L\right)$. Assume that we are given back-tracers $(t)$ for all atomic terms $t$. Suppose that $f$ and $g$ are maps from program points of $P$ to abstract values in $\mathcal{A}$ (i.e., $f, g \in \prod_{n \in V} \mathcal{A}$ ) such that $g$ approximates the abstract one-step execution from $f: F(f) \sqsubseteq g$ for the abstract step function $F$ for $P$. For such $f$ and $g$, we define the back-tracer $\left(|P|_{f g}\right.$ for $P$ to be the following function:

$$
\begin{aligned}
&(\mid P)_{f g}:\left(\prod_{n \in V} \mathcal{E}\right) \rightarrow\left(\prod_{n \in V} \mathcal{E}\right) \\
&(|P|)_{f g}(\epsilon)(n) \stackrel{\text { def }}{=} \prod\left\{\left(|L(n m)|_{f(n) g(m)}(\epsilon(m)) \mid n m \in E\right\},\right.
\end{aligned}
$$

where $\prod_{n \in V} \mathcal{E}$ is the cartesian product of lattices $\mathcal{E}$, ordered pointwise. We call $\epsilon \in \prod_{n \in V} \mathcal{E}$ extractor annotation. The back-tracer $\left(|P|_{f g}\right.$ for $P$ takes a post extractor annotation $\epsilon$ for $g$, and computes a pre extractor annotation $\epsilon^{\prime}$ for $f$, by first running given $(|L(n m)|)_{f(n) g(m)}$, and then combining all the resulting extractors at each program node.

Recall that back-tracers $(\mid L(n m))$ for atomic terms $L(n m)$ take only those subscripts $a b$ that satisfy $\llbracket L(n m) \rrbracket a \sqsubseteq b$. We note that when $(|P|)$ calls $\left(|L(n m)|_{f(n) g(m)}\right.$, it always uses correct subscripts; for each $n m \in E$,

$$
\begin{aligned}
\llbracket L(n m) \rrbracket f(n) & \sqsubseteq \bigsqcup\left\{\llbracket L\left(n^{\prime} m\right) \rrbracket f\left(n^{\prime}\right) \mid n^{\prime} m \in E\right\} & \text { (since } n m \in E \text { ) } \\
& =F(f)(m) & \text { (by the definition of } F \text { ) } \\
& \sqsubseteq g(m) & \text { (since } F(f) \sqsubseteq g) .
\end{aligned}
$$

We define exall to be the application of extractor annotations:

$$
\begin{aligned}
& \text { exall }:\left(\prod_{n \in V} \mathcal{E}\right) \rightarrow_{m}\left(\left(\prod_{n \in V} \mathcal{A}\right) \rightarrow_{m}\left(\prod_{n \in V} \mathcal{A}\right)\right) \\
& \operatorname{exall}(\epsilon)(f) \stackrel{\text { def }}{=} \lambda n \in V . \operatorname{ex}(\epsilon(n))(f(n)) .
\end{aligned}
$$

The following lemma shows that the back-tracer $(|P|)_{f g}$ computes a correct pre extractor annotation.

Lemma 3.9. For all $\epsilon$ in $\prod_{n \in V} \mathcal{E}$, if $\left(|P|_{f g}(\epsilon)=\epsilon^{\prime}\right.$,

$$
F\left(\operatorname{exall}\left(\epsilon^{\prime}\right)(f)\right) \sqsubseteq \operatorname{exall}(\epsilon)(g) .
$$

Proof. To show the lemma, pick an arbitrary program point $n$ from $V$. Then, for all $m$ such that $m n \in E$,

$$
\begin{array}{rlrl}
(\llbracket L(m n) \rrbracket f(m)) & \sqsubseteq\left(\bigsqcup_{m^{\prime} n \in E}\left(\llbracket L\left(m^{\prime} n\right) \rrbracket f\left(m^{\prime}\right)\right)\right) & \quad \text { (since } m n \in E) \\
& =(F(f)(n)) & & \text { (by the definition of } F) \\
& \sqsubseteq g(n) & \text { (by the assumption } F(f) \sqsubseteq g) .
\end{array}
$$


Let $e^{\prime}$ be $\left(|L(m n)|_{f(m) g(n)}(\epsilon(n))\right.$. By what we have derived above and the definition of back-tracers for $L(m n)$, we have that

$$
\llbracket L(m n) \rrbracket\left(\operatorname{ex}\left(e^{\prime}\right)(f(m))\right) \sqsubseteq \operatorname{ex}(\epsilon(n))(g(n)) .
$$

We now prove the required inequality as follows.

$$
\begin{aligned}
& F\left(\text { exall }\left((\mid P)_{f g}(\epsilon)\right)(f)\right)(n) \\
& \left.=\bigsqcup_{m n \in E}\left(\llbracket L(m n) \rrbracket\left(\left(\text { exall }\left((\mid P)_{f g}(\epsilon)\right)(f)\right)(m)\right)\right) \quad \text { (by the definition of } F\right) \\
& =\bigsqcup_{m n \in E}\left(\llbracket L(m n) \rrbracket\left(\operatorname{ex}\left((|P|)_{f g}(\epsilon)(m)\right)(f(m))\right)\right) \\
& \text { (since exall } \left.\left(\epsilon^{\prime}\right)(f)(m)=\operatorname{ex}\left(\epsilon^{\prime}(m)\right)(f(m)) \text { for all } \epsilon^{\prime}\right) \\
& =\bigsqcup_{m n \in E}\left(\llbracket L ( m n ) \rrbracket \left(\operatorname { e x } \left(\prod_{\left.\left.\left.\left\{\left(\left|L\left(m n^{\prime}\right)\right|\right)_{f(m) g\left(n^{\prime}\right)}\left(\epsilon\left(n^{\prime}\right)\right) \mid m n^{\prime} \in E\right\}\right)(f(m))\right)\right)}\right.\right.\right. \\
& \text { (by the definition of }\left(|P|_{f g}\right) \\
& \left.\sqsubseteq \bigsqcup_{m n \in E}\left(\llbracket L(m n) \rrbracket\left(\operatorname{ex}(\mid(L(m n)))_{f(m) g(n)}(\epsilon(n))\right)(f(m))\right)\right) \\
& \text { (since } m n \in E \text {, and } \llbracket L(m n) \rrbracket \text { and } \operatorname{ex}(-)(f(m)) \text { are monotone) } \\
& \sqsubseteq \bigsqcup_{m n \in E} \operatorname{ex}(\epsilon(n))(g(n)) \\
& \text { (since } \left.\forall e \in \mathcal{E} \text {. ex }(e)(g(n)) \sqsupseteq \llbracket L(m n) \rrbracket\left(\operatorname{ex}(|L(m n)|)_{f(m) g(n)}(e)\right)(f(m))\right) \\
& =\operatorname{ex}(\epsilon(n))(g(n)) \text {. }
\end{aligned}
$$

\subsection{Abstract-Value Slicer SL}

We now define an abstract-value slicer, assuming that we are given two components of the slicer, namely, an extractor domain $(\mathcal{E}$, ex) and back-tracers $(\mid-D$ for all atomic terms in this domain. Suppose that we are given a program $P=\left(V, E, n_{i}, n_{f}, L\right)$. Let $F$ be the abstract one-step execution of $P$ in the abstract interpretation, and let postfix $(F)$ be $\{f \mid F(f) \sqsubseteq f\}$, the set of post fixpoints of $F$.

Definition 3.10. The abstract-value slicer SL for the program $P$ is the function defined as follows:

$$
\begin{gathered}
\mathrm{SL}:\left(\operatorname{postfix}(F) \times \prod_{n \in V} \mathcal{E}\right) \rightarrow\left(\prod_{n \in V} \mathcal{E}\right) \\
\begin{aligned}
& \mathrm{SL}(f, \epsilon) \stackrel{\text { def }}{=} \text { let } B_{f}=\lambda \epsilon^{\prime} .\left(\epsilon^{\prime} \sqcap|P|_{f f} \epsilon^{\prime}\right) \text { and } \\
& k=\min \left\{n \mid n \geq 0 \wedge B_{f}^{n}(\epsilon)=B_{f}^{n+1}(\epsilon)\right\} \\
& \text { in } B_{f}^{k}(\epsilon) .
\end{aligned}
\end{gathered}
$$

Intuitively, the first input $f$ to the slicer denotes the result of the abstract interpretation, and the second $\epsilon$ specifies the part of $f$ that is used for verification; although exall $(\epsilon)(f)$ is weaker than $f$, it is still strong enough to verify the property of interest. Given such $f$ and $\epsilon$, the slicer SL defines a reductive 
function $^{14} B_{f}$ on $\prod_{n \in V} \mathcal{E}$, and then computes its fixpoint $\epsilon^{\prime}$ such that $\epsilon^{\prime} \sqsubseteq \epsilon$, by repeatedly applying $B_{f}$ from $\epsilon$. Note that SL always succeeds in computing such $\epsilon^{\prime}$, because the domain $\prod_{n \in V} \mathcal{E}$ of $B_{f}$ has finite height. The result of the slicer $\operatorname{SL}(f, \epsilon)$ is this computed fixpoint $\epsilon^{\prime} .^{15}$

The result $\operatorname{SL}(f, \epsilon)$ of the abstract-value slicer satisfies the following two important properties, which together ensure the correctness of the slicer:

(1) $\mathrm{SL}(f, \epsilon) \sqsubseteq \epsilon$, and

(2) exall(SL $(f, \epsilon))(f)$ is a post fixpoint of $F$.

The first property means that $\operatorname{SL}(f, \epsilon)$ extracts at least as much information as $\epsilon$, so that if a property of $P$ can be verified by exall $(\epsilon)(f)$, it can also be verified by exall $(\operatorname{SL}(f, \epsilon))(f)$. The second property means that exall $(\operatorname{SL}(f, \epsilon))(f)$ is another possible solution of the abstract interpretation, which could have been obtained if the abstract interpretation used a different strategy for computing post fixpoints. Note that the first property holds because $B_{f}$ is reductive and the fixpoint computation of the slicer starts from $\epsilon$. For the second property, we prove a slightly more general lemma, by using the soundness of the back-tracer $(|P|)$ (Lemma 3.9).

Lemma 3.11. For all $f \in \operatorname{postfix}(F)$ and all $\epsilon^{\prime} \in \prod_{n \in V} \mathcal{E}$,

$$
B_{f}\left(\epsilon^{\prime}\right)=\epsilon^{\prime} \Longrightarrow F\left(\operatorname{exall}\left(\epsilon^{\prime}\right)(f)\right) \sqsubseteq \operatorname{exall}\left(\epsilon^{\prime}\right)(f) .
$$

Proof. To show the lemma, choose an arbitrary post fixpoint $f$ of $F$ and an extractor annotation $\epsilon^{\prime}$ for $f$ such that $B_{f}\left(\epsilon^{\prime}\right)=\epsilon^{\prime}$. Then,

$$
\begin{aligned}
(\mid P)_{f f} \epsilon^{\prime} & \sqsupseteq B_{f}\left(\epsilon^{\prime}\right) & \text { (by the definition of } B_{f} \text { ) } \\
& =\epsilon^{\prime} & \text { (by assumption). }
\end{aligned}
$$

Using what we have just shown above, we prove the required inequality as follows:

exall $\left(\epsilon^{\prime}\right)(f) \sqsupseteq F\left(\operatorname{exall}\left((|P|)_{f f} \epsilon^{\prime}\right)(f)\right)$

(by Lemma 3.9)

$\sqsupseteq F\left(\right.$ exall $\left.\left(\epsilon^{\prime}\right)(f)\right)$ (since $\epsilon^{\prime} \sqsubseteq(|P|)_{f f} \epsilon^{\prime}$, and $F$ and exallare monotone).

We summarize what we have just proved in the following proposition.

Proposition 3.12 (Correctness). For all $f \in \operatorname{postfix}(F)$ and all $\epsilon \in \prod_{n \in V} \mathcal{E}$, the slicer $\operatorname{SL}(f, \epsilon)$ terminates (i.e., it is a well-defined total function), and it outputs $\epsilon^{\prime}$ such that $\epsilon^{\prime} \sqsubseteq \epsilon$ and $F\left(\operatorname{exall}\left(\epsilon^{\prime}\right)(f)\right) \sqsubseteq$ exall $\left(\epsilon^{\prime}\right)(f)$.

Example 3.13. Consider the following result from evenness analysis:

$$
\begin{aligned}
x & \mapsto \top_{e} \\
y & \mapsto \top_{e}
\end{aligned} \quad \mathrm{y}:=2 \mathrm{y} ; \begin{array}{ll}
x & \mapsto \top_{e} \\
y & \mapsto \text { even }
\end{array} \quad \mathrm{x}:=2 \mathrm{y} ; \begin{array}{ll}
x & \mapsto \text { even } \\
y & \mapsto \text { even }
\end{array} \mathrm{y}:=\mathrm{x}\left[\begin{array}{ll}
x & \mapsto \text { even } \\
y & \mapsto \text { even }
\end{array}\right.
$$

\footnotetext{
${ }^{14} \mathrm{~A}$ function $f$ on a poset $C$ is reductive iff $f(x) \sqsubseteq x$ for all $x \in C$.

${ }^{15}$ In general, the result of $\operatorname{SL}(f, \epsilon)$ is not the greatest fixpoint $\epsilon^{\prime}$ of $B_{f}$ satisfying the condition $\epsilon^{\prime} \sqsubseteq \epsilon$. In fact, such greatest fixpoints $\epsilon^{\prime}$ might not even exist. However, if $(|t|)_{a b}$ is monotone for all atomic terms $t$, so that $(\mid P)_{f g}$ is monotone, then the result of $\operatorname{SL}(f, \epsilon)$ is the greatest fixpoint $\epsilon^{\prime}$ of $B_{f}$ that satisfies the condition. In this case, the result of $S L(f, \epsilon)$ is the greatest fixpoint of $\lambda \epsilon^{\prime} . \epsilon \sqcap(P \mid) f f\left(\epsilon^{\prime}\right)$.
} 
Suppose that we have used the analysis in order to verify that variable y stores an even integer at the end. The following extractor annotation expresses this verification goal:

$$
\{\} \text { у }:=2 \mathrm{y} ;\{\} \mathrm{x}:=2 \mathrm{y} ;\{\} \text { y }:=\mathrm{x}\{y\}
$$

When the abstract-value slicer for evenness analysis is given the preceding analysis result and extractor annotation, it returns the extractor annotation following:

$$
\{\} \mathrm{y}:=2 \mathrm{y} ;\{\} \mathrm{x}:=2 \mathrm{y} ;\{x\} \text { y }:=\mathrm{x}\{y\}
$$

Thus, the original analysis result is sliced to:

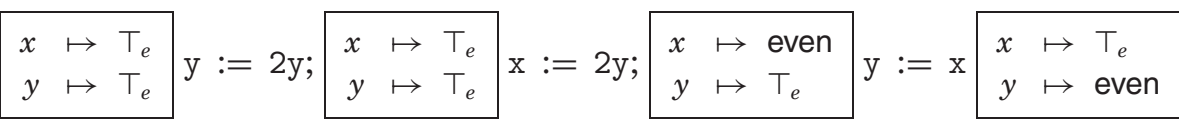

Note that the sliced result correctly expresses that the only necessary information for the verification is the evenness of $\mathrm{x}$ after $\mathrm{x}:=2 \mathrm{y}$ and the verification goal at the end.

Example 3.14. Figure 5 shows the result of the abstract-value slicing for zone analysis. Figure 5(b) shows the input to the slicer; the DBMs in the second row describe the result of zone analysis, and the extractors in the first row specify that only the $(2,1),(1,2)$-th entries of the DBM at $n_{4}$ are used for verification. Figure 5(c) shows the sliced result; the first row describes the result of the abstract-value slicer for this input, and the second row describes the application of the obtained extractors to the abstract interpretation result. For this example, this table indicates that among 19 non- $\infty$ DBM entries in the abstract interpretation result, only 5 entries are needed to prove the property of interest. Finally, Figure 5(d) expresses the abstract interpretation result and its slice in the form of constraints.

\section{METHODS FOR DESIGNING BACK-TRACERS FOR ATOMIC TERMS}

In this section, we provide two methods for designing back-tracers for atomic terms. As explained in Section 3.2, it is relatively easy to define a correct backtracer for an atomic term $t$. However, designing a good back-tracer for $t$ is difficult, and requires special knowledge about the abstract interpretation that is used. The first method in the section aims at producing accurate back-tracers for atomic terms: a slicer with the back-tracer that is produced usually filters out more information from the abstract interpretation result, than the one with naively designed back-tracers. The second method, on the other hand, aims at a back-tracer with low cost on time and space. Throughout the section, we assume a fixed abstract interpretation that uses an $\operatorname{abstract} \operatorname{domain}(\mathcal{A}, \sqsubseteq, \perp, \sqcup)$


extractor domain $(\mathcal{E}$, ex $)$ is given for this abstract interpretation, and that $\mathcal{E}$ is finite. 



(a) Program

\begin{tabular}{|c|c|c|c|c|c|c|c|c|c|c|}
\hline \multirow{2}{*}{$\begin{array}{c}\text { Initial extractor } \\
\text { annotation }\end{array}$} & \multirow{2}{*}{\multicolumn{2}{|c|}{$\begin{array}{l}n_{0} \\
\{\}\end{array}$}} & \multirow{2}{*}{\multicolumn{2}{|c|}{$\begin{array}{l}n_{1} \\
\{\}\end{array}$}} & \multirow{2}{*}{\multicolumn{2}{|c|}{$\begin{array}{l}n_{2} \\
\{\}\end{array}$}} & \multirow{2}{*}{\multicolumn{2}{|c|}{$\begin{array}{l}n_{3} \\
\{\}\end{array}$}} & \multicolumn{2}{|r|}{$n_{4}$} \\
\hline & & & & & & & & & $\{(1$, & $, 2),(2,1)\}$ \\
\hline & & $x_{0} x_{1} x_{2}$ & & $x_{0} x_{1} x_{2}$ & & $x_{0} x_{1} x_{2}$ & & $x_{0} x_{1} x_{2}$ & & $x_{0} x_{1} x_{2}$ \\
\hline $\begin{array}{l}\text { interpretation } \\
\quad \text { result }\end{array}$ & $\begin{array}{l}x_{0} \\
x_{1} \\
x_{2}\end{array}$ & $\begin{array}{lcc}\infty & 4 & 3 \\
-1 & \infty & \infty \\
-1 & 0 & \infty\end{array}$ & $\begin{array}{l}x_{0} \\
x_{1} \\
x_{2}\end{array}$ & $\begin{array}{llll}\infty & \infty & 3 \\
\infty & \infty & \infty \\
-1 & 1 & \infty\end{array}$ & $\begin{array}{l}x_{0} \\
x_{1} \\
x_{2}\end{array}$ & $\begin{array}{ccc}\infty & \infty & 3 \\
\infty & \infty & \infty \\
-1 & 0 & \infty\end{array}$ & $\begin{array}{l}x_{0} \\
x_{1} \\
x_{2}\end{array}$ & $\begin{array}{ccc}\infty & \infty & 3 \\
\infty & \infty & -1 \\
-1 & 1 & \infty\end{array}$ & $\begin{array}{l}x_{0} \\
x_{1} \\
x_{2}\end{array}$ & $\begin{array}{lll}\infty & \infty & 3 \\
\infty & \infty & -1 \\
-1 & 1 & \infty\end{array}$ \\
\hline
\end{tabular}

(b) Input arguments for abstract-value slicer

\begin{tabular}{|c|c|c|c|c|c|c|c|c|c|c|}
\hline & & $n_{0}$ & & $n_{1}$ & & $n_{2}$ & & $n_{3}$ & & $n_{4}$ \\
\hline $\begin{array}{l}\text { Result extractors from } \\
\text { abstract-value slicer }\end{array}$ & & $\{(2,1)\}$ & & $\{(2,1)\}$ & & \{\} & & $\{(2,1)\}$ & $\{(1$, & $, 2),(2,1)\}$ \\
\hline \multirow{4}{*}{$\begin{array}{c}\text { Sliced } \\
\text { abstract interpretation } \\
\text { result }\end{array}$} & & $x_{0} x_{1} x_{2}$ & & $x_{0} x_{1} x_{2}$ & & $x_{0} x_{1} x_{2}$ & & $x_{0} x_{1} x_{2}$ & & $x_{0} x_{1} x_{2}$ \\
\hline & $x_{0}$ & $\infty \infty \infty$ & $\omega_{0}$ & $\infty \infty \infty$ & $x_{0}$ & $\infty \infty \infty$ & $x_{0}$ & $\infty \infty \infty$ & $x_{0}$ & $\infty \infty \infty$ \\
\hline & & $\infty \infty \infty$ & $x_{1}$ & $\infty \infty \infty$ & $x_{1}$ & $\infty \infty \infty$ & $x_{1}$ & $\infty \infty \infty$ & $x_{1}$ & $\infty \infty-1$ \\
\hline & & $\infty \quad 0 \quad \infty$ & $x_{2}$ & $\infty 1 \infty$ & $x_{2}$ & $\infty \infty \infty$ & $x_{2}$ & $\infty 1 \infty$ & $x_{2}$ & $\infty 1 \infty$ \\
\hline
\end{tabular}

(c) Results from abstract-value slicer

\begin{tabular}{|c|c|c|c|c|c|}
\hline & $n_{0}$ & $n_{1}$ & $n_{2}$ & $n_{3}$ & $n_{4}$ \\
\hline $\begin{array}{c}\text { Before } \\
\text { slicing }\end{array}$ & $\begin{array}{c}1 \leq x_{1} \leq 4 \\
\wedge 1 \leq x_{2} \leq 3 \\
\wedge x_{1} \leq x_{2}\end{array}$ & $\begin{array}{c}1 \leq x_{2} \leq 3 \\
\wedge x_{1} \leq x_{2}+1\end{array}$ & $\begin{array}{c}1 \leq x_{2} \leq 3 \\
\wedge x_{1} \leq x_{2}\end{array}$ & $\begin{array}{l}1 \leq x_{2} \leq 3 \\
\wedge x_{1}=x_{2}+1\end{array}$ & $\begin{array}{c}1 \leq x_{2} \leq 3 \\
\wedge x_{1}=x_{2}+1\end{array}$ \\
\hline $\begin{array}{c}\text { After } \\
\text { slicing }\end{array}$ & $x_{1} \leq x_{2}$ & $x_{1} \leq x_{2}+1$ & & $x_{1} \leq x_{2}+1$ & $x_{1}=x_{2}+1$ \\
\hline
\end{tabular}

(d) Results as constraints

Fig. 5. Result of abstract-value slicing for the program in Figure 2.

\subsection{Best Back-Tracer Construction}

The first method constructs the best back-tracer for each atomic term, considered in Proposition 3.6. It is a slight modification of a rather well-known "reversing technique" [Hughes and Launchbury 1992; Duesterwald et al. 1995]. Let $t$ be an atomic term, and let $(a, b)$ be a pair of pre and post conditions in $\operatorname{prepost}(t)$. For these $t, a, b$, the method defines the back-tracer $(\mid t)_{a b}$ as follows:

$$
(|t|)_{a b}(e) \stackrel{\text { def }}{=} \bigsqcup\left\{e_{0} \in \mathcal{E} \mid \llbracket t \rrbracket\left(\operatorname{ex}\left(e_{0}\right)(a)\right) \sqsubseteq \operatorname{ex}(e)(b)\right\} .
$$


Intuitively, $(t t)_{a b}(e)$ is the "conjunction" of all correct pre extractors: $(|t|)_{a b}(e)$ selects some information from $a$, precisely when all the correct pre extractors select the same information. Note that for every correct pre extractor $e_{0}$, the computed extractor $e^{\prime}=(t)_{a b}(e)$ filters out at least as much information from $a$ as $e_{0}$ (i.e., $\left.\operatorname{ex}\left(e_{0}\right)(a) \sqsubseteq \operatorname{ex}\left(e^{\prime}\right)(a)\right)$, and so, it induces a better slice of $a$ than $e_{0}$.

In addition to the bestness of the method, we need to check whether the method constructs a correct back-tracer. Unfortunately, this method does not always construct a correct back-tracer; in general, the constructed $(|t|)_{a b}$ does not satisfy the following soundness condition from the definition of back-tracers:

$$
\forall e, e^{\prime} \in \mathcal{E} .(\mid t)_{a b}(e)=e^{\prime} \Longrightarrow \llbracket t \rrbracket\left(\operatorname{ex}\left(e^{\prime}\right)(a)\right) \sqsubseteq \operatorname{ex}(e)(b) .
$$

To make the above soundness condition hold, we should restrict the use of the method for join-preserving functions as the solutions in other studies [Hughes and Launchbury 1992; Duesterwald et al. 1995]: we use the constructed $\left(|t|_{a b}\right.$, only when $\llbracket t \rrbracket$ and $\operatorname{ex}(-)(a)$ preserve finite joins.

Lemma 4.1. If $\llbracket t \rrbracket$ and $\lambda$ e. $\operatorname{ex}(e)(a)$ preserve finite joins for all $a$, then $(t))$ satisfies the soundness condition for back-tracers for $t$. Moreover, in this case, $(\mid t)_{a b}$ is the best back-tracer for $t$ (which exists by Proposition 3.6).

PRoof. In this proof, we show that the method constructs a correct backtracer by the join-preservation, and then show why it is a best back-tracer. To see why the join-preservation provides a solution, suppose that $\llbracket t \rrbracket$ and ex $(-)(a)$ preserves finite joins. Then, their composition $\lambda e$. $\llbracket t \rrbracket(\operatorname{ex}(e)(a))$ also preserves finite joins. So, for all extractors $e, e^{\prime} \in \mathcal{E}$ such that $(t \mid)_{a b} e=e^{\prime}$, we have that

$$
\begin{aligned}
& \llbracket t \rrbracket\left(\operatorname{ex}\left(e^{\prime}\right)(a)\right) \\
& =\llbracket t \rrbracket\left(\operatorname{ex}\left((|t|)_{a b}(e)\right)(a)\right) \\
& \left.=\llbracket t \rrbracket\left(\operatorname{ex}\left(\bigsqcup\left\{e_{0} \mid \llbracket t \rrbracket\left(\operatorname{ex}\left(e_{0}\right)(a)\right) \sqsubseteq \operatorname{ex}(e)(b)\right\}\right)(a)\right) \quad \text { (by the definition of }(\mid t)_{a b}\right) \\
& =\left(\bigsqcup\left\{\llbracket t \rrbracket\left(\operatorname{ex}\left(e_{0}\right)(a)\right) \mid \llbracket t \rrbracket\left(\operatorname{ex}\left(e_{0}\right)(a)\right) \sqsubseteq \operatorname{ex}(e)(b)\right\}\right) \quad \text { (by join preservation) } \\
& \sqsubseteq \operatorname{ex}(e)(b) \text {. }
\end{aligned}
$$

This order relationship implies the correctness of $(\mid t)_{a b}$. Now, we prove that $(|t|)_{a b}$ is a best back-tracer. Consider a back-tracer $k$ for $t$. Then, for all $(a, b) \in$ $\operatorname{prepost}(t)$ and $e \in \mathcal{E}$,

$$
\llbracket t \rrbracket\left(\operatorname{ex}\left(k_{a b}(e)\right)(a)\right) \sqsubseteq \operatorname{ex}(e)(b) .
$$

Thus, by the definition of $(t t)$, we have that $k_{a b}(e) \sqsubseteq(t)_{a b}(e)$, as required.

The very definition of $(\mid t)_{a b}$ gives a default (usually inefficient) implementation, if all the extractor applications are computable. When a post extractor $e$ for $b$ is given, the implementation calculates all the correct pre extractors for $a, b, t, e$; this is possible, because the extractor domain $\mathcal{E}$ is finite (by assumption) and $\llbracket t \rrbracket$ is computable. Then, the implementation returns the greatest one from the calculated extractors. This default implementation is, however, very slow, and in many cases, it can be improved dramatically. We illustrate this improvement using zone analysis. 
Example 4.2. Consider zone analysis $(\mathcal{M}, \sqsubseteq, \perp, \sqcup)$ and the extractor domain $(\mathcal{E}$, ex) in Example 3.2. In this case, the method in this section can be applied to obtain the best back-tracer for an atomic term, if the term is either a Boolean expression or an assignment of the form $x_{i}:=x_{i}+c$; zone analysis interprets all such atomic terms as join-preserving functions, and extractor application ex $(-)(a)$ preserves finite joins for all $a$. In this example, we will explain how to efficiently implement this best back-tracer.

Let $t$ be an atomic term that is either a Boolean expression or an assignment of the form $x_{i}:=x_{i}+c$. Our implementation of the best back-tracer for $t$ is based on two important observations.

(1) First, no matter whether $t$ is a Boolean expression or an assignment, the abstract semantics $\llbracket t \rrbracket$ of $t$ is a pointwise transformation of DBM matrices; to compute the $i j$-th entry of the output DBM, $\llbracket t \rrbracket$ uses at most the $i j$-th entry of the input DBM. More precisely, there exists a family $\left\{f_{i j}\right\}_{i j \in(N+1) \times(N+1)}$ of monotone functions on Ints $\cup\{-\infty, \infty\}$ (ordered by $\leq$ ) such that

$$
\forall i j .(\llbracket t \rrbracket a)_{i j}=f_{i j}\left(a_{i j}\right) .
$$

(2) Second, when a function family $\left\{f_{i j}\right\}_{i j}$ determines $\llbracket t \rrbracket$, it can be used to simplify the "correctness condition" for pre and post extractors: for every $(a, b) \in \operatorname{prepost}(t)$, pre extractor $e_{0} \in \mathcal{E}$ and post extractor $e \in \mathcal{E}$, we have that

$\left(\llbracket t \rrbracket\left(\operatorname{ex}\left(e_{0}\right)(a)\right) \sqsubseteq \operatorname{ex}(e)(b)\right) \Longleftrightarrow\left(\forall i j . i j \in e \Rightarrow\left(i j \in e_{0} \vee f_{i j}(\infty) \leq b_{i j}\right)\right)$.

Intuitively, this simplified condition says that every index $i j$ in the post extractor $e$ should belong to the pre extractor $e_{0}$, except when $t$ can "generate" the information $b_{i j}$ (i.e., $x_{j}-x_{i} \leq b_{i j}$ ) without using the input DBM. To see this, note that since $f_{i j}$ is monotone, $f_{i j}(\infty) \leq b_{i j}$ implies that for every input DBM $a^{\prime}$, the $i j$-th entry of $\llbracket t \rrbracket\left(a^{\prime}\right)$ should be less than or equal to $b_{i j}$. Thus, no information from the input DBM is necessary for $t$ to "produce" the $i j$-th entry of $b$.

We now use these two observations to optimize the best back-tracer for $t$ :

$\bigsqcup\left\{e_{0} \mid \llbracket t \rrbracket\left(\operatorname{ex}\left(e_{0}\right)(a)\right) \sqsubseteq \operatorname{ex}(e)(b)\right\}$

$=\bigcap\left\{e_{0} \mid \llbracket t \rrbracket\left(\operatorname{ex}\left(e_{0}\right)(a)\right) \sqsubseteq \operatorname{ex}(e)(b)\right\} \quad$ (by the lattice structure of $\mathcal{E}$ )

$=\bigcap\left\{e_{0} \mid \forall i j . i j \in e \Rightarrow\left(i j \in e_{0} \vee f_{i j}(\infty) \leq b_{i j}\right)\right\} \quad$ (by the second observation)

$=\bigcap\left\{e_{0} \mid \forall i j .\left(i j \in e \wedge f_{i j}(\infty) \not \leq b_{i j}\right) \Rightarrow i j \in e_{0}\right\}$

$=\bigcap\left\{e_{0} \mid\left\{i j \mid i j \in e \wedge f_{i j}(\infty) \not \leq b_{i j}\right\} \subseteq e_{0}\right\}$

$=\left\{i j \mid i j \in e \wedge f_{i j}(\infty) \not \leq b_{i j}\right\}$

$=e-\left\{i j \mid f_{i j}(\infty) \leq b_{i j}\right\}$.

Note that the obtained formula indicates the efficient implementation of the best back-tracer $\left(\|\left. f\right|_{a b}\right.$ as a single set subtraction. Moreover, the subtracted set in the formula is a fixed set that does not depend on the post extractor $e$. This property can allow a further optimization of the set subtraction. In fact, the 
back-tracers for Boolean expressions $E \leq E^{\prime}$, assignments $x_{i}:=x_{i}+c$ and command skip in Figure 4 are such further optimizations.

Before finishing the discussion on the construction of best back-tracers, we consider one special case that the extractor domain is an atomic lattice. Recall (from the standard lattice theory [Davey and Priestley 1990]) that an element $x$ in a lattice $L$ is an atom if and only if it is the second smallest element in $L$ :

$$
\left.x \neq \perp \wedge\left(\forall x^{\prime} \in L .\left(x^{\prime} \sqsubseteq x \wedge x^{\prime} \neq x\right) \Rightarrow x^{\prime}=\perp\right)\right),
$$

and that a lattice $L$ is atomic if and only if every element $x$ in the lattice $L$ can be reconstructed by combining (by join) all the atoms $x^{\prime}$ such that $x^{\prime} \sqsubseteq x$ :

$$
\forall x \in L . x=\bigsqcup\left\{x^{\prime} \mid x^{\prime} \sqsubseteq x \text { and } x^{\prime} \text { is an atom }\right\} .
$$

The following proposition suggests that we can optimize the best back-tracer $(t t)_{a b}$ when $\mathcal{E}$ is atomic.

Proposition 4.3. When $\mathcal{E}$ is atomic, the best back-tracer $(|t|)_{a b}$ is identical to the following function:

$$
\lambda e . \bigsqcup\left\{e_{0} \in \mathcal{E} \mid \llbracket t \rrbracket\left(\operatorname{ex}\left(e_{0}\right)(a)\right) \sqsubseteq \operatorname{ex}(e)(b) \text { and } e_{0} \text { is an atom }\right\} .
$$

Proof. Let $k_{a b}$ be the function defined in the proposition. For all $e_{0} \in$ $\left\{e_{0} \in \mathcal{E} \mid \llbracket t \rrbracket\left(\operatorname{ex}\left(e_{0}\right)(a)\right) \sqsubseteq \operatorname{ex}(e)(b)\right.$ and $e_{0}$ is an atom\}, it follows $e_{0} \sqsubseteq\left(\left.t\right|_{a b}(e)\right.$ by Lemma 3.5. Since $k_{a b}(e)$ is the join of all such $e_{0}$, we have that $k_{a b}(e) \sqsubseteq(\mid t)_{a b}(e)$. For the other direction, consider an arbitrary $e_{1} \in \mathcal{E}$ such that

$$
\llbracket t \rrbracket\left(\operatorname{ex}\left(e_{1}\right)(a)\right) \sqsubseteq \operatorname{ex}(e)(b) .
$$

Note that such $e_{1}$ can be $(t)_{a b}$ by the definition (Definition 3.3) of back-tracer. For all atoms $e_{0}$ such that $e_{0} \sqsubseteq e_{1}$,

$$
\begin{array}{rlrl}
\llbracket t \rrbracket\left(\operatorname{ex}\left(e_{0}\right)(a)\right) & \left.\sqsubseteq \llbracket t \rrbracket\left(\operatorname{ex}\left(e_{1}\right)(a)\right) \quad \text { (by the monotonicity of } \llbracket f \rrbracket \text { and ex }(-)(a)\right) \\
& \sqsubseteq \operatorname{ex}(e)(b) & \text { (by the choice of } \left.e_{1}\right) .
\end{array}
$$

Thus, $e_{0} \sqsubseteq k_{a b}(e)$. This implies that $e_{1} \sqsubseteq k_{a b}(e)$ since $\mathcal{E}$ is atomic. So, $\left(|t|_{a b}(e) \sqsubseteq\right.$ $k_{a b}(e)$.

\subsection{Extension Method}

The second method, called extension method, is a dual approach to the atomic lattice case considered in the previous section. Extension method assumes two properties of the extractor domain. Recall (from the standard lattice theory [Davey and Priestley 1990]) that an element $x$ in a lattice $L$ is a dual atom if and only if it is the second biggest element in $L$ :

$$
\left.x \neq \top \wedge\left(\forall x^{\prime} \in L .\left(x \sqsubseteq x^{\prime} \wedge x^{\prime} \neq x\right) \Rightarrow x^{\prime}=\top\right)\right),
$$

and that a lattice $L$ is dual atomic if and only if every element $x$ in the lattice $L$ can be reconstructed by combining (by meet) all the dual atoms $x^{\prime}$ such that $x \sqsubseteq x^{\prime}$ :

$$
\forall x \in L . x=\prod\left\{x^{\prime} \mid x \sqsubseteq x^{\prime} \text { and } x^{\prime} \text { is a dual atom }\right\} .
$$


The first assumption of the extension method is that each extractor lattice $\mathcal{E}$ is dual atomic, and the second assumption is that each extractor application preserves all finite meets:

$$
\operatorname{ex}(\top)=\top \quad \text { and } \quad \operatorname{ex}\left(e \sqcap e^{\prime}\right)=\operatorname{ex}(e) \sqcap \operatorname{ex}\left(e^{\prime}\right) .
$$

Here $\top$ and $\sqcap$ are the lattice operations for upper closure operators, and they are defined pointwise. ${ }^{16}$ Intuitively, these two assumptions mean that every extractor $e$ in $\mathcal{E}$ represents a collection $\left\{e_{1}, \ldots, e_{n}\right\}$ of dual atomic extractors; $e$ extracts information from $a$ by first applying each $e_{i}$ to $a$ and then conjoining all the resulting information:

$$
\operatorname{ex}(e)(a)=\prod_{i=1, \ldots, n} \operatorname{ex}\left(e_{i}\right)(a) .
$$

When the extractor domain satisfies the preceding assumptions, the extension method provides a recipe for constructing correct back-tracers for all atomic terms. Let $t$ be an atomic term and $(a, b)$ a pair of pre and post conditions in $\operatorname{prepost}(t)$. The first step of the extension method is to define a partial backtracer $g: g$ is a partial function of type $\mathcal{E} \rightarrow \mathcal{E}$ such that (1) the domain of $g$ is precisely the set of dual atoms in $\mathcal{E}$ and (2) for all post extractors in $\operatorname{dom}(g), g$ calculates correct pre extractors:

$$
\forall e \in \operatorname{dom}(g) . \llbracket t \rrbracket(\operatorname{ex}(g(e))(a)) \sqsubseteq \operatorname{ex}(e)(b) .
$$

The next step is to extend $g$ to the following complete back-tracer:

$$
\left.(t)_{a b}(e) \stackrel{\text { def }}{=}\right\rceil\left\{g\left(e_{1}\right) \mid e \sqsubseteq e_{1} \text { and } e_{1} \text { is a dual atom }\right\} .
$$

The total back-tracer $(t)_{a b}(e)$ decomposes the post extractor $e$ into dual atoms, and then applies $g$ to all the obtained dual atoms; finally, it merges all the resulting pre extractors (by meet). Note that, since $\mathcal{E}$ is finite, the meet here is over the finite sets, and so, it is well defined. The following lemma shows that the constructed $(t)_{a b}$ is correct.

Lemma 4.4. For all extractors $e, e^{\prime} \in \mathcal{E}$, we have that

$$
e^{\prime}=\left(|t|_{a b}(e) \Longrightarrow \llbracket t \rrbracket\left(\operatorname{ex}\left(e^{\prime}\right)(a)\right) \sqsubseteq \operatorname{ex}(e)(b) .\right.
$$

PRoof. We prove the lemma as follows:

$$
\begin{aligned}
& \llbracket t \rrbracket\left(\operatorname{ex}\left((t \mid)_{a b} e\right)(a)\right) \\
& \left.=\llbracket t \rrbracket\left(\operatorname{ex}\left(\prod_{\{g}\left(e_{1}\right) \mid e \sqsubseteq e_{1} \text { and } e_{1} \text { is a dual atom }\right\}\right)(a)\right) \\
& \text { (by the definition of } \left.(t)_{a b}\right) \\
& =\llbracket t \rrbracket\left(\prod_{\left.\left\{\operatorname{ex}\left(g\left(e_{1}\right)\right)(a) \mid e \sqsubseteq e_{1} \text { and } e_{1} \text { is a dual atom }\right\}\right)}\right. \\
& \text { (by the meet preservation of ex) } \\
& \sqsubseteq \prod\left\{\llbracket t \rrbracket\left(\operatorname{ex}\left(g\left(e_{1}\right)\right)(a)\right) \mid e \sqsubseteq e_{1} \text { and } e_{1} \text { is a dual atom }\right\} \quad \text { (since } \llbracket t \rrbracket \text { is monotone) } \\
& \sqsubseteq \prod\left\{\operatorname{ex}\left(e_{1}\right)(b) \mid e \sqsubseteq e_{1} \text { and } e_{1} \text { is a dual atom }\right\} \\
& \text { (since } \left.\llbracket t \rrbracket\left(\operatorname{ex}\left(g\left(e_{1}\right)\right)(a)\right) \sqsubseteq \operatorname{ex}\left(e_{1}\right)(b) \text { for all dual atoms } e_{1}\right)
\end{aligned}
$$

\footnotetext{
${ }^{16}$ Precisely, $\top(a)=\top$ and $\left(\operatorname{ex}(e) \sqcap \operatorname{ex}\left(e^{\prime}\right)\right)(a)=\operatorname{ex}(e)(a) \sqcap \operatorname{ex}\left(e^{\prime}\right)(a)$.
} 




(by the meet preservation of ex)

$=\operatorname{ex}(e)(b)$

(since $\mathcal{E}$ is dual atomic).

The extension method has two advantages over the best back-tracer construction. First, the extension method usually provides a relatively efficient default implementation of the defined back-tracers. By "efficient," we do not mean a linear-time algorithm, but simply mean a polynomial-time algorithm, instead of exponential-time algorithm. Suppose that we have defined a backtracer $(t t)_{a b}$, by applying the extension method to a partial back-tracer $g$. The default implementation of $\left(|t|_{a b}\right.$ uses an internal table $T$ that records the graph of function $g$, and is defined as follows:

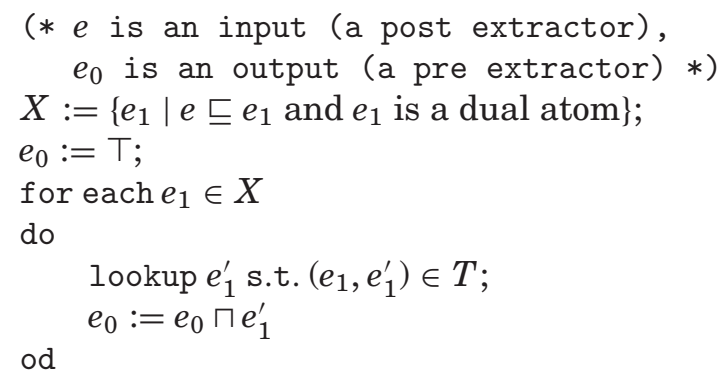

Here we specify the implementation imperatively, in order to distinguish it from the definition of $\left(|t|_{a b}\right.$. Note that this implementation simply follows the definition of $(|t|)_{a b}$ without any special optimizations. However, the implementation is efficient. In the worst case, the set $X$ contains all the dual atoms, and so, all the basic operations in the implementation, such as the look-up of table $T$ and the meet operation of extractors, are executed at most as many times as the number of dual atoms in $\mathcal{E}$. Even when the extractor lattice $\mathcal{E}$ is big, they contain only smaller number of dual atoms; in many cases, even though the cardinality of an extractor lattice is exponential in the size of the program, the number of dual atoms in the lattice is polynomial in the program size. Thus, in such cases, the implementation runs in polynomial time. Note that in the best back-tracer construction, a naive implementation, which directly follows the definition, executes basic operations as many times as the size of the extractor lattice.

Second, the extension method is more widely applicable than the best backtracer construction. The assumptions of the extension method are only about the extractor domain, not about the abstract interpretation. Thus, once the extractor domain is well chosen, the method can be used to get back-tracers for all atomic terms. This contrasts with the best back-tracer construction, which can be applicable to an atomic term $t$ only when $\llbracket t \rrbracket$ preserves finite joins.

Example 4.5. For zone analysis and the extractor domain ( $\mathcal{E}$, ex) in Example 3.2, the extension method can be applied to all atomic terms; $\mathcal{E}$ is a dual atomic lattice, whose dual atoms are singleton index sets $\{i j\}$, and the extractor 
application ex preserves finite meets. Here we apply the method to construct back-tracers for assignments $x_{i}:=E$ that were not handled in Example 4.2, namely those that are not of the form $x_{i}:=x_{i}+c$. In fact, for such assignments $x_{i}:=E$, we cannot apply the best back-tracer construction, because $\llbracket x_{i}:=E \rrbracket$ does not preserve some finite joins.

Let $a, b$ be DBMs such that $\llbracket x_{i}:=E \rrbracket a \sqsubseteq b$. To apply the extension method, we need to define a correct partial back-tracer $g$ for $x_{i}:=E$ at $a$ and $b$. We define such $g$ by doing the case analysis on the input DBM $a$. When $a$ contains a cycle with negative weight, we pick one such cycle pickNegCycle $(a)=k_{0} k_{1} \ldots k_{n}$ in $a$, and define $g$ to be a constant function $\lambda e .\left\{k_{0} k_{1}, k_{1} k_{2}, \ldots, k_{n-1} k_{n}\right\}$. Otherwise, that is, when $a$ does not contain a negative cycle, we define $g$ as follows, using paths with minimum weight:

$$
\begin{aligned}
g(\{k l\}) \stackrel{\text { def }}{=} & \text { if }\left(b_{k l}=\infty \vee k=i \vee l=i\right) \\
& \text { then }\{\} \\
& \text { else } \left.\left(\text { if } a_{k l} \leq b_{k l} \text { then }\{k l\} \text { else edges(mPath }(a, k, l)\right)\right) .
\end{aligned}
$$

Here we used the subroutine edges in Figure 4, which takes a path $k_{0} k_{1} \ldots k_{n}$ and returns the set $\left\{k_{0} k_{1}, k_{1} k_{2}, \ldots, k_{n-1} k_{n}\right\}$ of all the edges in the path. The defined function $g$ first checks whether $\llbracket t \rrbracket$ needs to use the input to generate $b_{k l}$. If so, $g$ returns the entries of the input $a$ that are necessary for generating $b_{k l}$. Otherwise, $g$ returns the empty set.

Now the extension method gives the back-tracer $\left(\left|x_{i}:=E\right|\right)_{a b}$ defined as follows:

$$
\left(\mid x_{i}:=E\right)_{a b}(e) \stackrel{\text { def }}{=} \prod\left\{g\left(e_{1}\right) \mid e \sqsubseteq e_{1} \text { and } e_{1} \text { is a dual atom }\right\} .
$$

This definition can be optimized to the back-tracer for $x_{i}:=E$ in Figure 4 . When a contains a negative cycle,

$$
\begin{aligned}
& \left(\mid x_{i}:=E\right)_{a b}(e) \\
& =\prod\left\{g\left(e_{1}\right) \mid e \sqsubseteq e_{1} \text { and } e_{1} \text { is a dual atom }\right\} \\
& =\prod\left\{\text { edges }(\operatorname{pickNegCycle}(a)) \mid e \sqsubseteq e_{1} \text { and } e_{1} \text { is a dual atom }\right\} \\
& \text { (by the definition of } g \text { ) } \\
& =\text { edges }(\operatorname{pickNegCycle}(a)) \text {. }
\end{aligned}
$$

When $a$ does not contain a negative cycle,

$$
\begin{aligned}
&\left(x_{i}\right.:=\left.E\right|_{a b}(e) \\
&= \prod\left\{g\left(e_{1}\right) \mid e \sqsubseteq e_{1} \text { and } e_{1} \text { is a dual atom }\right\} \\
&=\left.\bigcup\left\{g\left(e_{1}\right) \mid e \sqsubseteq e_{1} \text { and } e_{1} \text { is a dual atom }\right\} \quad \text { (by the lattice structure of } \mathcal{E}\right) \\
&= \bigcup_{k l \in e} g(\{k l\}) \\
& \quad\left(\text { ince }\left(e_{1}=\{k l\} \text { for some } k l \in e\right) \Longleftrightarrow\left(e \sqsubseteq e_{1} \text { and } e_{1} \text { is a dual atom) }\right)\right. \\
&= \bigcup_{k l \in e} \text { if } \operatorname{then}\left\{b_{k l}=\infty \vee i=k \vee i=l\right) \\
& \quad \text { else }\left(\text { if } a_{k l} \leq b_{k l} \text { then }\{k l\} \text { else edges }(\operatorname{mPath}(a, k, l))\right)
\end{aligned}
$$

(by the definition of $g$ ) 


$$
\begin{aligned}
= & \text { let } e^{\prime}=e-\left\{k l \mid b_{k l}=\infty \vee k=i \vee l=i\right\} \\
& \text { in } \left.\bigcup_{k l \in e^{\prime}} \text { if } a_{k l} \leq b_{k l} \text { then }\{k l\} \text { else edges(mPath }(a, k, l)\right) \\
= & \text { let } e^{\prime}=e-\left\{k l \mid b_{k l}=\infty\right\}-\{i k, k i \mid 0 \leq k \leq N\} \\
& \text { in } \left.\bigcup_{k l \in e^{\prime}} \text { if } a_{k l} \leq b_{k l} \text { then }\{k l\} \text { else edges(mPath }(a, k, l)\right) .
\end{aligned}
$$

Note that in both cases, the optimized definition coincides with the back-tracer for $\left(\left|x_{k}:=E\right|\right)$ in Figure 4.

\section{EXPERIMENTS}

We designed an abstract-value slicer for the full zone analysis [Miné 2001], and tested the efficiency of the resulting slicer in the context of proof generation.

\subsection{Abstract-Value Slicer for the Full Zone Analysis}

The full zone analysis is different from our simplified version in Example 2.2 in two aspects. First, the full analysis additionally applies the DBM closure operator -* (in Example 2.2) before all DBM joins in the analysis. Second, it has better abstract semantics of atomic terms. For all the assignments $x_{i}:=$ $E$, if $E$ does not have the form $c, x_{i}+c$, or $x_{j}+c$, our simplified analysis replaces $x_{i}:=E$ by a random assignment $x_{i}:=$, which chooses an integer nondeterministically and assigns the chosen number to $x_{i}$; then, the simplified analysis defines $\llbracket x_{i}:=E \rrbracket$ to be the strongest postcondition transformer of $x_{i}:=$ ?. The full version, on the other hand, does not do such a replacement, but defines more accurate abstract semantics of $x_{i}:=E$ using interval analysis. Given an input DBM $a$, the full analysis first applies the closure $-{ }^{*}$ to $a$, just like the simplified analysis. But then, instead of updating all $x_{i}$-related entries by $\infty$, the full analysis estimates the range of the right-hand side expression $E$ of $x_{i}:=E$, using interval analysis. It projects $a^{*}$ into the following abstract value $\operatorname{prj}\left(a^{*}\right)$ in interval analysis

$$
\operatorname{prj}\left(a^{*}\right)=\lambda x_{j} \cdot\left[-\left(a^{*}\right)_{j 0},\left(a^{*}\right)_{0 j}\right],
$$

and runs $\llbracket E \rrbracket\left(\operatorname{prj}\left(a^{*}\right)\right)$ in interval analysis to obtain the (approximate) range $[n, m]$ of $E$. Finally, using this obtained range of $E$, the full analysis updates the $i 0$ and $0 i$ entries of the input $a^{*}$, and returns the following DBM:

$$
\left(\left(a^{*}\right)[k i \mapsto \infty, i k \mapsto \infty]_{1 \leq k \neq i \leq N}\right)[0 i \mapsto m, i 0 \mapsto-n] .
$$

We designed an abstract-value slicer for the full zone analysis, by modifying the slicer for the simplified version in Examples 3.2 and 3.8. To deal with the additional uses of the closure operator $-^{*}$, we defined the back-tracer $\beta$ for -* as follows. For all $a, b$ such that $a^{*} \sqsubseteq b$,

$$
\begin{aligned}
\beta_{a b}: & \mathcal{E} \rightarrow \mathcal{E} \\
\beta_{a b}(e) \stackrel{\text { def }}{=} & \text { if }(\text { hasNegCycle }(a)=\text { true }) \\
& \text { then edges }(\operatorname{pickNegCycle}(a)) \\
& \text { else let } e^{\prime}=e-\left\{k l \mid b_{k l}=\infty\right\} \\
\quad & \text { in } \bigcup_{k l \in e^{\prime}}\left(\text { if } a_{k l} \leq b_{k l} \text { then }\{k l\} \text { else edges }(\operatorname{mPath}(a, k, l))\right) .
\end{aligned}
$$


Table I. Number of Sliced DBM Entries

\begin{tabular}{|l|c|c|c|c|c|}
\hline \multirow{2}{*}{ Program } & \multicolumn{3}{|c|}{ Number of DBM entries $^{*}$} & \multirow{2}{*}{ Slicing time } \\
\cline { 2 - 5 } & (1)total $^{a}$ & Extracted $^{b}$ & (2)removed $^{c}$ & $(2) /(1)$ & Slion \\
\hline \hline Insertionsort & 92 & 22 & 70 & $76 \%$ & 0.07 \\
\hline Partition $^{d}$ & 120 & 46 & 74 & $62 \%$ & 0.03 \\
\hline Bubblesort & 217 & 42 & 175 & $81 \%$ & 0.11 \\
\hline KMP $^{e}$ & 463 & 133 & 330 & $72 \%$ & 0.28 \\
\hline Heapsort & 817 & 181 & 636 & $78 \%$ & 0.29 \\
\hline
\end{tabular}

${ }^{a}$ Number of non- $\infty$ DBM entries in the results of zone analysis.

${ }^{b}$ Number of the DBM entries in (1) that are extracted (i.e., not changed) by the slicer.

${ }^{c}$ Number of the DBM entries in (1) that are changed to $\infty$ by the slicer.

${ }^{d}$ Partition function in Quicksort.

${ }^{e}$ Knuth-Morris-Pratt pattern matching algorithm.

The defined $\beta$ was, then, inserted into the old slicer in Example 3.8, in order to back-trace newly added closure applications in the full analysis. To handle the modified abstract semantics $\llbracket x:=E \rrbracket$, we designed a slicer for interval analysis, which contains a back-tracer $(|E|)$ for expression $E$. Then, using $(|E|)$, we changed the old back-tracer $(|x:=E|)$, in order to account for the new improved abstract semantics of $x:=E$.

\subsection{Experimental Results}

We implemented the full zone analysis, the abstract-value slicer, and the proof construction algorithm using our previous work [Seo et al. 2003]. In our experiment, we first executed the analysis with five array accessing programs, and obtained approximate invariants which are strong enough to show the absence of array bounds errors. Then, we ran the slicer for each of the computed abstract interpretation results, and measured the number of invariants (i.e., DBM entries) in the results that have been eliminated (i.e., replaced by $\infty$ ). Finally, we applied the proof construction algorithm to both the original abstract interpretation results and their sliced versions, and measured how much the slicer reduced the size of the constructed proofs.

Table I shows the number of invariants that have been sliced out by the abstract-value slicer. The second column, labeled by "total," contains the number of all the nontrivial DBM entries (i.e., entries that are not $\infty$ ) in the result of the abstract interpreter, and the fourth column, labeled by "removed," shows how many of those nontrivial entries the slicer found unnecessary for verifying the absence of array bounds errors. The experimental result shows that about $62 \%$ to $81 \%$ of computed invariants are not needed for the verification.

The reduction in the size of constructed proofs is shown in Table II. The constructed proofs are trees whose nodes express the application of Hoare logic rule or first-order logic rule. The nodes for first-order logic rules have different sizes, depending on the first-order logic formulas that are contained in the nodes. Thus, for each constructed proof, we counted three entities: the nodes for Hoare logic rules, the nodes for first-order logic rules, and the first-order formulas. The abstract-value slicer did not reduce the number of Hoare logic 
Table II. Reduction in the Proof Size

\begin{tabular}{|c|c|c|c|c|c|c|}
\hline \multirow[b]{2}{*}{ Program } & \multicolumn{2}{|c|}{ Before slicing } & \multicolumn{2}{|c|}{ After slicing } & \multirow[b]{2}{*}{$(1)-(3) /(1)$} & \multirow{2}{*}{$\begin{array}{c}\text { Reduction in } \\
\text { proof size }\end{array}$} \\
\hline & $(1) \mathrm{FOL}^{a}$ & (2)formulas ${ }^{b}$ & $(3) \mathrm{FOL}^{c}$ & (4)formulas ${ }^{d}$ & & \\
\hline Insertionsort & 248 & 2530 & 166 & 1122 & $33 \%$ & $53 \%$ \\
\hline Partition & 398 & 3866 & 201 & 1847 & $49 \%$ & $52 \%$ \\
\hline Bubblesort & 894 & 12230 & 389 & 2677 & $56 \%$ & $76 \%$ \\
\hline KMP & 1364 & 26898 & 653 & 7683 & $52 \%$ & $70 \%$ \\
\hline Heapsort & 2542 & 52370 & 1028 & 7936 & $60 \%$ & $84 \%$ \\
\hline
\end{tabular}

${ }^{a}$ Number of nodes for first-order logic rules that appear in the proof tree for an original (unsliced) analysis result. ${ }^{b}$ Number of first-order formulas that appear in the proof tree for the original analysis result.

${ }^{c}$ Number of nodes for first-order logic rules that appear in the proof tree for a sliced analysis result.

${ }^{d}$ Number of formulas that appear in the proof tree for the sliced analysis result.

${ }^{e}$ Here the size of a proof counts all of applied Hoare logic rules, applied first-order logic rules, and first-order logic formulas in the proof.

rules, because Hoare rules are applied as many times as the number of program constructs in the program, and the abstract-value slicer does not change the program. However, the slicer reduced the number of first-order logic rules and the number of first-order formulas. In Table II, we show those numbers before and after slicing. The experimental result shows that in the proof trees for sliced analysis results, about 33\% to 60\% less rules are used for showing implications between first-order logic formulas. In the seventh column of the table, we show the reduction ratio in the size of the whole proofs. For each of the constructed proof trees, we add the number of the nodes and that of first-order formulas, and then, we compute the reduction ratio in this number. The experimental result shows that the proof trees for sliced analysis results are about $52 \%$ to $84 \%$ smaller than those for original analysis results.

\section{CONCLUSION}

In this paper, we have presented a framework for abstract-value slicers that weaken the abstract interpretation results. We have presented two design guides to define back-tracers for atomic terms that propagate the slicing information of each atomic term backwards. In fact, designing a back-tracer is a key task in implementing an abstract-value slicer.

The motivating application of the slicer is to reduce the proof size in the proof construction method [Seo et al. 2003] that takes the program invariants computed by an abstract interpretation and produces a Hoare proof for these invariants. Since the slicer reduces the number of invariants to prove, it enables us to have smaller proofs. In our experiment in constructing the proofs for the absence of array bound violations in five small yet representative array-access programs, our slicing algorithm reduce the proofs' sizes. In our experiment with zone analysis, the slicer identified $62 \%-81 \%$ of the abstract interpretation results as unnecessary, and resulted in 52\%-84\% reduction in the proof size.

Our abstract-value slicer has been targeted for one specific application, the construction of Hoare proofs from abstract interpretation results. For instance, our slicer guarantees that the sliced analysis results are post fixpoints of the abstract transfer function. Because of this guarantee, the following proofconstruction phase does not have to call a (possibly expensive) theorem prover, 
but it can instead rely on the soundness of the abstract interpretation only [Seo et al. 2003].

While this paper was being reviewed, Besson et al. [2007] have independently considered the problem of weakening abstraction interpretation results and developed an approach similar to our abstract value slicer. However, the focus of their work is slightly different from ours. Their work emphasizes the issue of the existence of the weakest abstract interpretation results that prove the property of interest. On the other hand, this paper focuses on algorithms for weakening abstract interpretation results and a general framework for validating the soundness of such algorithms.

One interesting future direction is to disconnect the tie between the proof construction and our framework for abstract-value slicers, and revisit the framework. For instance, instead of asking the sliced analysis results to be post fixpoints of abstract transfer functions, we might require them to be post fixpoints of concrete transfer functions. This might lead to a new formulation of abstractvalue slicers, which is suitable for studying semantics-driven slicing.

Abstract-value slicers can be seen as algorithms for simplifying an abstract domain without losing the abstract-interpretation based proof of a property of interest. Concretely, consider a join semi lattice $\mathcal{D}$, a monotone function $F: \mathcal{D} \rightarrow_{m}$ $\mathcal{D}$, and abstract values $d_{0}, d \in \mathcal{D}$, such that

$$
d_{0} \sqsubseteq d \text { and } F\left(d_{0}\right) \sqsubseteq d_{0} .
$$

Here $\mathcal{D}$ represents an abstract domain for an entire program (not for a single program point) and $F$ an abstract transfer function. Abstract values $d_{0}$ and $d$ denote an abstract-interpretation result and a property to verify, respectively, ${ }^{17}$ and the condition on $d_{0}$ and $d$ means that the abstract interpreter is able to prove $d$. In this setting, an abstract-value slicer can be considered to compute an upper closure operator $\rho$ on $\mathcal{D}$, such that

$$
\left.\rho\left(d_{0}\right) \sqsubseteq d \text { and }(\rho \circ F)\left(\rho\left(d_{0}\right)\right) \sqsubseteq \rho\left(d_{0}\right) \quad \text { (equivalently, } F\left(\rho\left(d_{0}\right)\right) \sqsubseteq \rho\left(d_{0}\right)\right) .
$$

That is, it simplifies the abstract domain $\mathcal{D}$ to $\rho(\mathcal{D})$, such that the induced best abstract transfer function $\rho \circ F$ in the simplified domain can still verify the property $d$, using $\rho\left(d_{0}\right)$. Moreover, the slicer attempts to make $\rho$ as abstract as possible.

The question about simplifying or compressing abstract domains has already been studied in the theory of abstract domain transformations [Filé et al. 1996; Giacobazzi and Ranzato 1997; Giacobazzi et al. 2000; Cortesi et al. 1998]. It would be interesting to see how the existing results can be used to give a new insight for designing better abstract value slicers. We currently expect that the work on compressing abstract domains can answer when the most abstract (i.e., biggest) upper closure operator $\rho$ satisfying the condition in the previous paragraph exists.

Finally, another interesting future direction is to develop more recipes for building abstract-value slicers. In particular, by using the known techniques for

${ }^{17}$ Domain $\mathcal{D}$ amounts to $\prod_{n \in V} \mathcal{A}$ in Section 2 , and $d_{0}$ and $d$ correspond to $f$ and exall $\left(\epsilon_{0}, f\right)$ in Definition 3.10. 
constructing abstract interpretations systematically [Cousot and Cousot 1979; Giacobazzi et al. 2000; Giacobazzi and Ranzato 1999; Giacobazzi and Scozzari 1998], we can provide corresponding systematic methods for building abstractvalue slicers. Some preliminary results in this direction appear in Yang et al. [2006].

\section{ACKNOWLEDGMENTS}

We would like to thank David Schmidt, Alan Mycroft, Xavier Rival, Daejun Park and anonymous referees for their helpful comments.

\section{REFERENCES}

Appel, A. W. 2001. Foundational proof-carrying code. In Proceedings of the IEEE Symposium on Logic in Computer Science (LICS). IEEE Computer Society Press, Los Alamitos, 247258.

Appel, A. W. AND Felty, A. P. 2000. A semantic model of types and machine instructions for proof-carrying code. In Proceedings of the ACM SIGPLAN-SIGACT Symposium on Principles of Programming Languages (POPL). ACM Press, New York, 243-253.

Ball, T., Majumdar, R., Millstein, T., and Rajamani, S. K. 2001. Automatic predicate abstraction of C programs. In Proceedings of the ACM SIGPLAN Conference on Programming Language Design and Implementation (PLDI). ACM Press, New York, 203-213.

BaLl, T. AND RAJAMANI, S. K. 2001. Automatically validating temporal safety properties of interfaces. In Proceedings of the SPIN Workshop on Model Checking of Software. Lecture Notes in Computer Science (LNCS), vol. 2057. Springer-Verlag, 103-122.

Besson, F., Jensen, T., And Turphin, T. 2007. Small witnesses for abstract interpretation-based proofs. In Proceedings of the European Symposium on Programming (ESOP). Lecture Notes in Computer Science, vol. 4421. Springer-Verlag, 268-283.

Bourdoncle, F. 1993. Abstract debugging of higher-order imperative languages. In Proceedings of the ACM SIGPLAN Conference on Programming Language Design and Implementation (PLDI). ACM Press, New York, 46-55.

Clarke, E. M., Grumberg, O., Jha, S., Lu, Y., and Veith, H. 2000. Counterexample-Guided abstraction refinement. In Proceedings of the International Conference on Computer-Aided Verification $(C A V)$. Lecture Notes in Computer Science, vol. 1855. Springer-Verlag, 154169.

Clarke, E. M., Grumberg, O., and Peled, D. A. 1999. Model Checking. The MiT Press.

Cortesi, A., Filé, G., AND Winsborough, W. H. 1998. The quotient of an abstract interpretation. Theor. Comput. Sci. 202, 1-2, 163-192.

Cousot, P. 1981. Semantic foundations of program analysis. In Program Flow Analysis: Theory and Applications, S. Muchnick and N. Jones, Eds. Prentice-Hall, Inc., Englewood Cliffs, NJ, Chapter 10, 303-342.

Cousot, P. 1998. The calculational design of a generic abstract interpreter. In Course notes for the NATO International Summer School Marktoberdorf (Germany) on Calculational System Design, M. Broy and R. Steinbrüggen, Eds. NATO ASI Series F. IOS Press, Amsterdam.

Cousot, P. 2005. Abstract interpretation. MIT course 16.399, http://web.mit.edu/16. 399/www/.

Cousot, P. And Cousot, R. 1977. Abstract interpretation: A unified lattice model for static analysis of programs by construction or approximation of fixpoints. In Proceedings of the ACM SIGPLAN-SIGACT Symposium on Principles of Programming Languages (POPL). ACM Press, New York, 238-252.

Cousot, P. And Cousot, R. 1979. Systematic design of program analysis frameworks. In Proceedings of the ACM SIGPLAN-SIGACT Symposium on Principles of Programming Languages (POPL). ACM Press, New York, 269-282.

Cousot, P. And Cousot, R. 1999. Refining model checking by abstract interpretation. Autom. Softw. Engin. 6, 1, 69-95. 
Dams, D., Gerth, R., And Grumberg, O. 1997. Abstract interpretation of reactive systems. ACM Trans. Program. Lang. Syst. 19, 2, 253-291.

Davey, D. A. And Priestley, H. A. 1990. Introduction to Lattices and Order. Cambridge University Press.

Davis, K. And Wadler, P. L. $\quad$ 1990. Backwards strictness analysis: Proved and improved. In Functional Programming: Proceedings of the 1989 Glasgow Workshop. Springer-Verlag, 12-30.

Duesterwald, E., Gupta, R., AND Soffa, M. L. 1995. Demand-driven computation of interprocedural data flow. In Proceedings of the ACM SIGPLAN-SIGACT Symposium on Principles of Programming Languages (POPL). ACM Press, New York, 37-48.

Filé, G., Giacobazzi, R., And Ranzato, F. 1996. A unifying view of abstract domain design. ACM Comput. Surv. 28, 2, 333-336.

GiacobazZi, R. ANd Mastroeni, I. 2004. Abstract noninterference: parameterizing noninterference by abstract interpretation. In Proceedings of the ACM SIGPLAN-SIGACT Symposium on Principles of Programming Languages (POPL). ACM Press, New York, 186-197.

Giacobazzi, R. AND Ranzato, F. 1997. Refining and compressing abstract domains. In Proceedings of the International Colloquium on Automata, Languages and Programming (ICALP). Lecture Notes in Computer Science, vol. 1256. Springer-Verlag, 771-781.

Giacobazzi, R. And Ranzato, F. 1999. The reduced relative power operation on abstract domains. Theor. Comput. Sci. 216, 1-2, 159-211.

Giacobazzi, R., Ranzato, F., AND Scozzari, F. 2000. Making abstract interpretations complete. J. ACM 47, 2, 361-416.

GiAcobAZZI, R. AND ScozZARI, F. 1998. A logical model for relational abstract domains. ACM Trans. Program. Lang. Syst. 20, 5, 1067-1109.

GRAF, S. AND SAÏDI, H. 1997. Construction of abstract state graphs with pvs. In Proceedings of the International Conference on Computer Aided Verification (CAV). Lecture Notes in Computer Science, vol. 1254. Springer-Verlag, 72-83.

Hamid, N., Shaoi, Z., Trifonov, V., Monnier, S., And Ni, Z. 2002. A syntactic approach to foundational proof-carrying code. In Proceedings of the IEEE Symposium on Logic in Computer Science (LICS). IEEE Computer Society Press, Los Alamitos, 89-100.

Henzinger, T., Jhala, R., Majumdar, R., and Sutre, G. $\quad 2002$. Lazy abstraction. In Proceedings of the ACM SIGPLAN-SIGACT Symposium on Principles of Programming Languages (POPL). ACM Press, New York, 58-70.

Henzinger, T., Jhala, R., Majumdar, R., and Sutre, G. 2003. Software verification with blast. In Proceedings of the SPIN Workshop on Model Checking of Software. Lecture Notes in Computer Science, vol. 2648. Springer-Verlag, 235-239.

HoARe, C. A. R. 1969. An axiomatic basis for computer programming. Comm. ACM 12, 10, 576580.

Howe, J. M., KING, A., AND Lu, L. 2004. Analysing logic programs by reasoning backwards. In Program Development in Computational Logic. Lecture Notes in Computer Science, vol. 3049. Springer-Verlag, 152-188.

Hughes, J. 1988. Backwards analysis of functional programs. In Proceedings of the IFIP TC2 Workshop on Partial Evaluation and Mixed Computation. Elsevier, 187-208.

Hughes, J. And Launchbury, J. 1992. Reversing abstract interpretations. In Proceedings of the European Symposium on Programming (ESOP). Lecture Notes in Computer Science, vol. 582. Springer-Verlag, 269-286.

KInG, A. AND LU, L. 2002. A backward analysis for constraint logic programs. Theory Prac. Logic Progr. 2, 4-5, 517-547.

Massé, D. 2001. Combining forward and backward analyses of temporal properties. In Proceedings of the 2nd Symposium on Programs as Data Objects (PADO). Lecture Notes in Computer Science, vol. 2053. Springer-Verlag, 103-116.

Miné, A. 2001. A new numerical abstract domain based on difference-bound matrices. In Proceedings of the 2nd Symposium on Programs as Data Objects (PADO). Lecture Notes in Computer Science, vol. 2053. Springer-Verlag, 155-172.

Morrisett, G., Walker, D., Crary, K., and Glew, N. 1998. From System F to typed assembly language. In Proceedings of the ACM SIGPLAN-SIGACT Symposium on Principles of Programming Languages (POPL). ACM Press, New York, 85-97. 
Necula, G. C. 1997. Proof-carrying code. In Proceedings of the ACM SIGPLAN-SIGACT Symposium on Principles of Programming Languages (POPL). ACM Press, New York, 106-119.

Necula, G. C. And Lee, P. 1997. Safe, untrusted agents using proof-carrying code. In Special Issue on Mobile Agent Security, G. Vigna, Ed. Lecture Notes in Computer Science, vol. 1419. Springer-Verlag, 61-91.

NeCULA, G. C. ANd RahUl, S. P. 2001. Oracle-based checking of untrusted software. In Proceedings of the ACM SIGPLAN-SIGACT Symposium on Principles of Programming Languages (POPL). ACM Press, New York, 142-154.

Necula, G. C. AND Schneck, R. 2002. Proof-carrying code with untrusted proof rules. In Software Security-Theories and Systems. Lecture Notes in Computer Science, vol. 2609. Springer-Verlag, $283-298$.

RIVAL, X. 2005a. Abstract dependences for alarm diagnosis. In Proceedings of the Asian Symposium on Programming Languages and Systems (APLAS). Lecture Notes in Computer Science, vol. 3780. Springer-Verlag, 347-363.

RIVAL, X. 2005b. Understanding the origin of alarms in ASTRÉE. In Proceedings of the International Static Analysis Symposium (SAS). Lecture Notes in Computer Science, vol. 3672. Springer-Verlag, 303-319.

Seo, S., Yang, H., AND YI, K. 2003. Automatic construction of Hoare proofs from abstract interpretation results. In Proceedings of the Asian Symposium on Programming Languages and Systems (APLAS). Lecture Notes in Computer Science, vol. 2895. Springer-Verlag, 230-245.

TIP, F. $\quad$ 1995. A survey of program slicing techniques. J. Program. Lang. 3, 3, 121-189.

Wadler, P. And Hughes, R. J. M. 1987. Projections for Strictness Analysis. In Functional Programming Languages and Computer Architecture, G. Kahn, Ed. Lecture Notes in Computer Science, vol. 274. Springer, Berlin, 385-407.

YAnG, H., Seo, S., YI, K., AND Han, T. 2006. Off-line semantic slicing from abstract interpretation results. Tech. mem. ROPAS-2006-34, Programming Research Laboratory, School of Computer Science \& Engineering, Seoul National University. Available at http://ropas. snu.ac.kr/lib/dock/YaSeYiHa2006.pdf.

Received December 2005; revised October 2006; accepted May 2007 